\begin{tabular}{|c|c|c|c|c|c|c|c|c|c|c|c|c|c|c|c|}
\hline & \multicolumn{7}{|c|}{ Unadjusted $^{5}$} & \multicolumn{7}{|c|}{ Seasonally adjusted and temperature corrected (annual rate) ${ }^{6}$} \\
\hline & & \multirow[b]{2}{*}{ Coal $^{1}$} & \multirow[b]{2}{*}{$\begin{array}{l}\text { Petro- } \\
\text { leum }^{2}\end{array}$} & \multirow[b]{2}{*}{$\begin{array}{c}\text { Natural } \\
\text { gas }^{3}\end{array}$} & \multicolumn{3}{|c|}{ Primary electricity } & Total & & & & Prim & ary electr & icity & Total \\
\hline & & & & & Nuclear & $\begin{array}{c}\text { Natural } \\
\text { flow } \\
\text { hydro }\end{array}$ & $\begin{array}{c}\text { Net } \\
\text { imports }\end{array}$ & & Coal & $\begin{array}{l}\text { Petro- } \\
\text { leum }\end{array}$ & $\begin{array}{c}\text { Natural } \\
\text { gas }\end{array}$ & Nuclear & $\begin{array}{c}\text { Natural } \\
\text { flow } \\
\text { hydro }\end{array}$ & \begin{tabular}{|c|} 
Net \\
imports
\end{tabular} & \\
\hline & & BHBB & $\mathrm{BHBC}$ & BHBD & BHBE & $\mathrm{BHBF}$ & BHBM & BHBA & $\mathrm{BHBH}$ & $\mathrm{BHBI}$ & BHBJ & BHBK & BHBL & $\mathrm{BHBN}$ & BHBG \\
\hline 2002 & & 39.3 & 73.5 & 95.4 & 20.1 & 0.5 & 0.7 & 229.5 & 40.2 & 74.9 & 100.9 & 20.1 & 0.5 & 0.7 & 237.3 \\
\hline 2003 & & 41.9 & 73.0 & 95.9 & 20.0 & 0.4 & 0.2 & 231.4 & 42.7 & 73.9 & 100.3 & 20.0 & 0.4 & 0.2 & 237.5 \\
\hline 2004 & & 41.0 & 75.1 & 98.1 & 18.2 & 0.6 & 0.6 & 233.6 & 41.7 & 76.1 & 103.0 & 18.2 & 0.6 & 0.6 & 240.2 \\
\hline 2005 & & 42.3 & 77.0 & 95.8 & 18.4 & 0.7 & 0.7 & 234.9 & 43.0 & 77.6 & 99.1 & 18.4 & 0.7 & 0.7 & 239.6 \\
\hline 2006 & & 46.1 & 76.3 & 91.1 & 17.1 & 0.8 & 0.6 & 232.0 & 47.0 & 77.4 & 92.9 & 17.1 & 0.8 & 0.6 & 235.8 \\
\hline 2005 & & 42.3 & 77.0 & 95.8 & 18.4 & 0.7 & 0.7 & 234.9 & 43.0 & 77.6 & 99.1 & 18.4 & 0.7 & 0.7 & 239.6 \\
\hline 2006 & & 46.1 & 76.3 & 91.1 & 17.1 & 0.8 & 0.6 & 232.0 & 47.0 & 77.4 & 92.9 & 17.1 & 0.8 & 0.6 & 235.8 \\
\hline 2007 & & 43.9 & 76.2 & 92.0 & 14.0 & 0.9 & 0.4 & 227.5 & 44.7 & 77.4 & 95.0 & 14.0 & 0.9 & 0.4 & 232.5 \\
\hline 2008 & & 41.4 & 75.1 & 94.9 & 11.9 & 1.1 & 0.9 & 225.2 & 41.6 & 75.4 & 95.4 & 11.9 & 1.1 & 0.9 & 226.3 \\
\hline 2009 & & 35.2 & 71.4 & 87.8 & 15.2 & 1.3 & 0.2 & 211.1 & 35.4 & 71.8 & 88.7 & 15.2 & 1.3 & 0.2 & 212.6 \\
\hline 2006 & Jan & 4.9 & 6.2 & 10.8 & 1.8 & 0.1 & 0.1 & 23.8 & 49.7 & 72.5 & 90.7 & 19.5 & 0.6 & 0.7 & 233.6 \\
\hline & Feb & 4.5 & 6.2 & 10.0 & 1.5 & 0.1 & 0.0 & 22.3 & 48.6 & 79.2 & 88.6 & 18.3 & 0.6 & 0.2 & 235.5 \\
\hline & Mar & 5.0 & 7.3 & 10.3 & 1.8 & 0.1 & 0.0 & 24.4 & 49.8 & 82.5 & 91.6 & 20.0 & 0.6 & 0.5 & 245.0 \\
\hline & April & 3.3 & 6.0 & 7.9 & 1.6 & 0.1 & 0.1 & 19.0 & 44.7 & 72.1 & 92.5 & 19.9 & 0.8 & 1.2 & 231.2 \\
\hline & May & 3.4 & 6.8 & 6.3 & 1.5 & 0.1 & 0.1 & 18.1 & 50.2 & 79.7 & 92.3 & 19.2 & 0.8 & 1.2 & 243.4 \\
\hline & June & 3.0 & 6.0 & 5.1 & 1.4 & 0.0 & 0.0 & 15.5 & 46.1 & 76.7 & 92.8 & 16.6 & 0.8 & 0.5 & 233.5 \\
\hline & July & 3.2 & 6.5 & 4.6 & 1.4 & 0.0 & 0.0 & 16.0 & 52.7 & 79.4 & 89.1 & 16.9 & 0.7 & 0.5 & 239.3 \\
\hline & Aug & 2.9 & 6.4 & 4.9 & 1.5 & 0.0 & 0.1 & 15.9 & 45.5 & 77.8 & 91.0 & 18.4 & 0.7 & 0.7 & 234.1 \\
\hline & Sep & 3.1 & 6.1 & 5.3 & 1.3 & 0.1 & 0.0 & 15.8 & 43.8 & 76.8 & 96.4 & 15.2 & 0.8 & 0.4 & 233.4 \\
\hline & Oct & 3.3 & 6.1 & 7.0 & 1.0 & 0.1 & 0.1 & 17.5 & 42.6 & 79.3 & 97.5 & 13.8 & 0.8 & 0.8 & 234.8 \\
\hline & Nov & 4.5 & 6.8 & 8.8 & 1.1 & 0.1 & 0.1 & 21.3 & 44.5 & 81.6 & 93.6 & 13.8 & 0.9 & 0.7 & 235.1 \\
\hline & Dec & 4.6 & 6.6 & 9.9 & 1.2 & 0.1 & 0.0 & 22.5 & 42.6 & 79.9 & 94.2 & 13.9 & 1.0 & 0.2 & 231.8 \\
\hline 2007 & Jan & 4.6 & 6.6 & 10.2 & 1.2 & 0.1 & 0.0 & 22.8 & 48.3 & 79.9 & 94.2 & 13.7 & 0.9 & 0.3 & 237.4 \\
\hline & Feb & 3.6 & 5.5 & 9.7 & 1.1 & 0.1 & 0.0 & 20.1 & 41.0 & 73.6 & 96.2 & 13.1 & 0.8 & 0.3 & 225.0 \\
\hline & Mar & 3.8 & 6.6 & 9.8 & 1.1 & 0.1 & 0.0 & 21.5 & 40.4 & 76.9 & 98.8 & 12.9 & 1.0 & 0.6 & 230.6 \\
\hline & April & 2.8 & 6.1 & 7.2 & 1.1 & 0.1 & 0.0 & 17.3 & 39.6 & 77.3 & 97.5 & 13.6 & 0.8 & -0.1 & 228.6 \\
\hline & May & 3.2 & 6.8 & 6.7 & 1.1 & 0.1 & 0.0 & 17.9 & 48.1 & 81.5 & 95.2 & 13.3 & 0.9 & 0.3 & 239.2 \\
\hline & June & 2.8 & 6.0 & 5.6 & 1.2 & 0.1 & 0.1 & 15.6 & 41.6 & 76.1 & 96.7 & 14.4 & 0.9 & 0.7 & 230.4 \\
\hline & July & 3.1 & 6.4 & 5.1 & 1.4 & 0.1 & 0.1 & 16.1 & 45.2 & 75.8 & 92.8 & 15.7 & 1.0 & 0.8 & 231.4 \\
\hline & Aug & 3.0 & 6.7 & 5.0 & 1.2 & 0.1 & 0.1 & 16.1 & 45.9 & 79.9 & 92.3 & 14.4 & 1.0 & 1.1 & 234.6 \\
\hline & Sept & 3.2 & 6.3 & 5.6 & 1.3 & 0.1 & 0.1 & 16.4 & 43.6 & 75.6 & 92.2 & 14.8 & 0.9 & 0.8 & 228.0 \\
\hline & Oct & 4.0 & 5.9 & 7.4 & 1.1 & 0.1 & 0.0 & 18.5 & 49.3 & 74.2 & 95.7 & 15.1 & 0.8 & 0.4 & 235.4 \\
\hline & Nov & 4.5 & 6.3 & 9.0 & 1.1 & 0.1 & 0.0 & 21.0 & 44.5 & 75.1 & 94.2 & 14.2 & 0.9 & 0.4 & 229.1 \\
\hline & Dec & 4.9 & 6.2 & 10.5 & 1.1 & 0.1 & 0.0 & 22.8 & 44.5 & 75.0 & 94.7 & 12.6 & 0.9 & -0.1 & 227.7 \\
\hline 2008 & Jan & 4.0 & 6.4 & 10.8 & 1.1 & 0.1 & 0.1 & 22.5 & 40.8 & 77.4 & 99.2 & 12.3 & 0.9 & 1.0 & 231.8 \\
\hline & Feb & 3.6 & 5.9 & 10.2 & 1.1 & 0.1 & 0.1 & 21.0 & 40.3 & 78.3 & 100.0 & 13.0 & 1.1 & 0.8 & 233.6 \\
\hline & Mar & 3.8 & 6.5 & 10.3 & 1.0 & 0.1 & 0.1 & 21.8 & 41.2 & 74.4 & 99.7 & 11.2 & 1.1 & 0.7 & 228.3 \\
\hline & Apr & 3.8 & 6.7 & 8.5 & 0.8 & 0.1 & 0.1 & 19.9 & 53.7 & 78.6 & 97.3 & 9.7 & 1.1 & 0.9 & 241.4 \\
\hline & May & 2.8 & 6.1 & 6.3 & 1.0 & 0.1 & 0.1 & 16.4 & 42.5 & 72.8 & 96.4 & 12.4 & 0.9 & 1.4 & 226.6 \\
\hline & June & 2.9 & 5.9 & 5.5 & 1.0 & 0.1 & 0.1 & 15.4 & 42.7 & 74.0 & 94.5 & 11.6 & 1.0 & 1.3 & 225.1 \\
\hline & July & 2.8 & 5.9 & 5.4 & 0.9 & 0.1 & 0.1 & 15.2 & 41.1 & 72.5 & 95.5 & 10.4 & 1.0 & 1.4 & 221.9 \\
\hline & Aug & 2.3 & 6.2 & 5.4 & 1.0 & 0.1 & 0.1 & 15.0 & 36.5 & 73.4 & 96.1 & 11.6 & 1.1 & 1.3 & 220.0 \\
\hline & Sep & 3.1 & 6.4 & 5.8 & 0.9 & 0.1 & 0.1 & 16.3 & 41.9 & 75.8 & 93.4 & 10.8 & 1.0 & 1.1 & 224.0 \\
\hline & Oct & 3.6 & 6.3 & 7.7 & 1.0 & 0.1 & 0.0 & 18.8 & 41.7 & 76.6 & 94.1 & 13.1 & 1.1 & 0.6 & 227.2 \\
\hline & Nov & 3.9 & 5.9 & 8.8 & 1.0 & 0.1 & 0.0 & 19.8 & 38.7 & 69.8 & 89.9 & 12.6 & 1.1 & 0.6 & 212.7 \\
\hline & Dec $^{*}$ & 4.4 & 5.9 & 10.2 & 1.2 & 0.1 & 0.0 & 21.8 & 38.4 & 70.7 & 88.8 & 13.8 & 1.1 & 0.2 & 212.3 \\
\hline 2009 & Jan & 4.7 & 6.2 & 10.8 & 1.0 & 0.1 & 0.0 & 22.8 & 41.5 & 70.3 & 88.1 & 15.3 & 1.1 & 0.1 & 212.5 \\
\hline & Feb & 4.0 & 5.6 & 9.2 & 1.3 & 0.1 & 0.0 & 20.2 & 44.1 & 72.6 & 86.7 & 17.2 & 0.9 & 0.2 & 217.0 \\
\hline & Mar & 3.5 & 6.5 & 8.5 & 1.5 & 0.1 & 0.0 & 20.2 & 38.2 & 74.6 & 86.8 & 17.2 & 1.1 & 0.3 & 218.1 \\
\hline & Apr & 2.6 & 6.0 & 6.5 & 1.5 & 0.1 & 0.1 & 16.7 & 37.5 & 72.0 & 84.7 & 17.6 & 1.2 & 0.9 & 213.8 \\
\hline & May & 2.5 & 5.8 & 5.9 & 1.2 & 0.1 & 0.1 & 15.7 & 35.7 & 69.8 & 86.1 & 15.3 & 1.3 & 1.3 & 209.5 \\
\hline & June & 2.4 & 5.6 & 5.0 & 1.4 & 0.1 & 0.1 & 14.6 & 36.6 & 72.0 & 85.6 & 16.1 & 1.2 & 0.7 & 212.2 \\
\hline & July & 2.2 & 5.9 & 4.9 & 1.4 & 0.1 & 0.1 & 14.5 & 33.3 & 71.9 & 87.7 & 15.9 & 1.2 & 0.7 & 210.6 \\
\hline & Aug & 2.0 & 6.3 & 4.9 & 1.3 & 0.1 & 0.0 & 14.7 & 33.1 & 73.8 & 89.1 & 15.8 & 1.4 & 0.4 & 213.6 \\
\hline & Sep & 2.3 & 5.7 & 5.6 & 1.2 & 0.1 & 0.0 & 14.8 & 32.9 & 68.8 & 91.6 & 14.8 & 1.4 & -0.2 & 209.2 \\
\hline & Oct & 3.0 & 6.0 & 6.9 & 1.1 & 0.1 & -0.1 & 17.0 & 35.2 & 72.4 & 91.4 & 15.1 & 1.1 & -0.7 & 214.5 \\
\hline & Nov & 2.8 & 5.9 & 8.6 & 1.2 & 0.1 & 0.0 & 18.6 & 29.7 & 71.5 & 93.7 & 15.7 & 1.3 & -0.3 & 211.7 \\
\hline & Dec & 3.4 & 5.6 & 11.0 & 1.3 & 0.1 & 0.0 & 21.4 & 31.5 & 67.9 & 95.8 & 14.7 & 1.1 & -0.3 & 210.8 \\
\hline 2010 & Jan & 4.0 & 5.7 & 11.9 & 1.4 & 0.1 & -0.1 & 23.1 & 34.2 & 64.8 & 95.1 & 15.3 & 0.9 & -0.8 & 209.5 \\
\hline & Feb & 3.6 & 5.6 & 10.4 & 1.2 & 0.1 & 0.0 & 20.9 & 36.2 & 70.5 & 92.2 & 14.1 & 0.8 & -0.6 & 213.3 \\
\hline & Mar & 3.0 & 6.2 & 9.9 & 1.4 & 0.1 & 0.0 & 20.5 & 33.4 & 69.7 & 97.4 & 15.2 & 0.9 & -0.4 & 216.2 \\
\hline & Apr & 2.5 & 5.7 & 7.5 & 1.1 & 0.1 & 0.0 & 16.9 & 36.1 & 67.6 & 93.0 & 13.6 & 1.2 & 0.0 & 211.6 \\
\hline & May & 2.5 & 5.9 & 6.8 & 1.0 & 0.1 & 0.0 & 16.2 & 35.3 & 69.2 & 94.1 & 13.0 & 1.0 & 0.4 & 213.0 \\
\hline & June & 2.4 & 5.6 & 5.2 & 0.9 & 0.1 & 0.1 & 14.3 & 36.4 & 71.9 & 87.4 & 11.1 & 1.1 & 1.0 & 208.7 \\
\hline & July & 2.6 & 5.9 & 4.8 & 1.0 & 0.1 & 0.1 & 14.5 & 38.5 & 73.2 & 89.1 & 11.1 & 1.7 & 1.0 & 214.5 \\
\hline & Aug & 2.2 & 6.1 & 5.0 & 1.0 & 0.1 & 0.1 & 14.5 & 35.3 & 70.4 & 89.5 & 11.6 & 1.3 & 1.3 & 209.4 \\
\hline
\end{tabular}

1 Include solid renewable sources (wood, straw and waste), a small amount of renewable primary heat sources (solar, geothermal, etc), and net foreign trade and stock changes in other solid fuels.

2 Inland deliveries for energy use, plus refinery fuel and losses, minus the differences between deliveries and actual consumption at power stations.

3 Includes gas used during production, colliery methane, landfill gas and sewage gas. Excludes gas flared or re-injected and non-energy use of gas

4 Includes generation. Excludes generation from pumped storage stations by solar pv.

5 Not seasonally adjusted or temperature corrected.

6 Coal, petroleum and natural gas are temperature corrected.

* 5 week month

Source: Department of Energy \& Climate Change 03000685056 


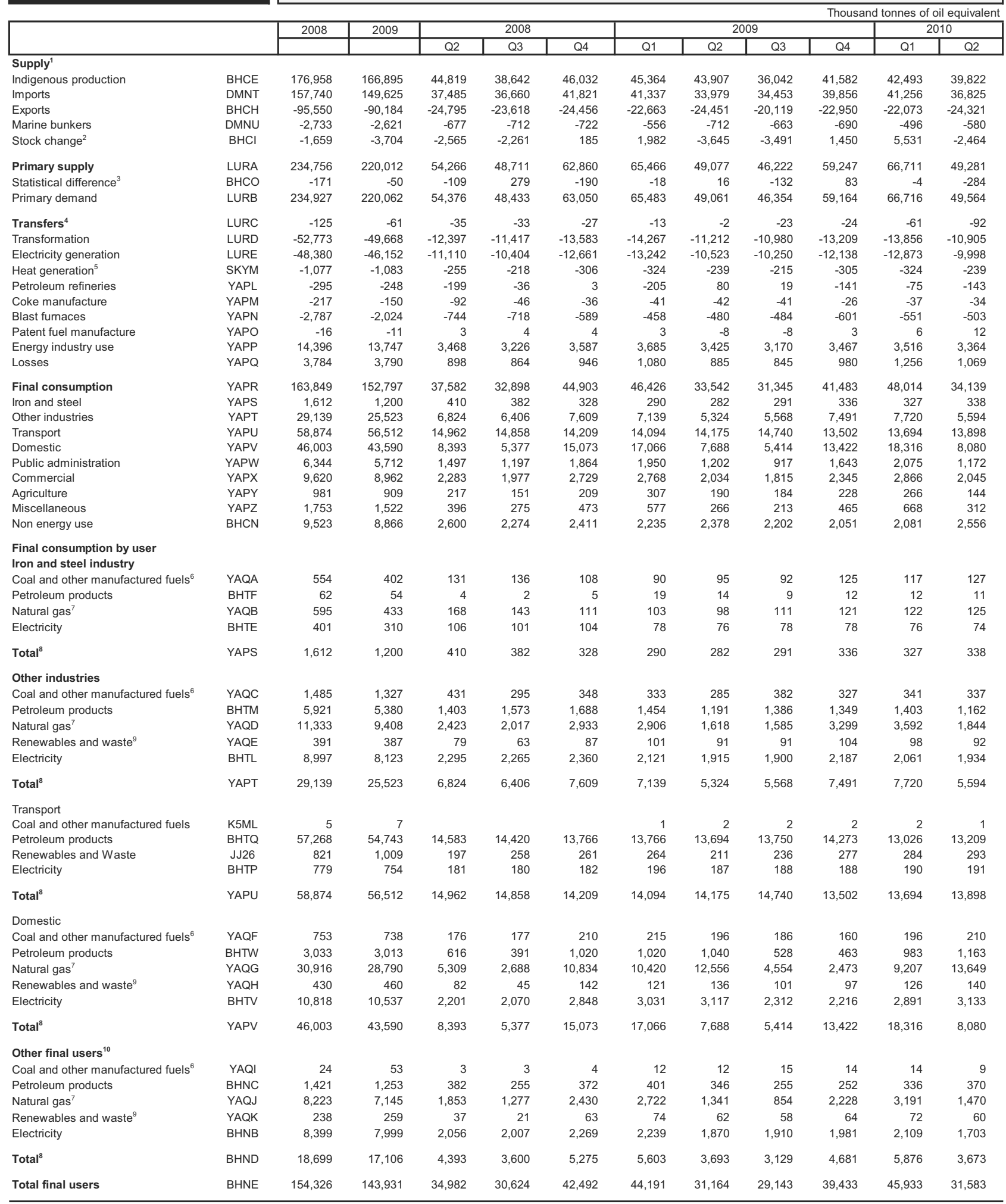

1 Layout comparable with annual balances published in Table 1.1 of DUKES 2006.

2 Stock fall (+), stock rise $(-)$.

3 Primary supply minus primary demand.

4 Annual transfers should ideally be zero. For manufactured fuels differences occur in the rescreening of coke to breeze. For oil and petroleum products differences arise due to

small variations in the calorific values used.

5 Generation of heat for sale under the provision of a contract.

6 Includes all manufactured solid fuels, benzole, tars, coke oven gas and blast furnace gas.

7 Includes colliery methane.

8 Includes heat sold.

9 Includes geothermal and solar heat. Latest quarter is estimated from the previous year and adjusted according to average annual rate of change over the last three years.

10 Includes public administration, commercial, agriculture and miscellaneous use.

Source: Department of Energy \& Climate Change 03000685056 


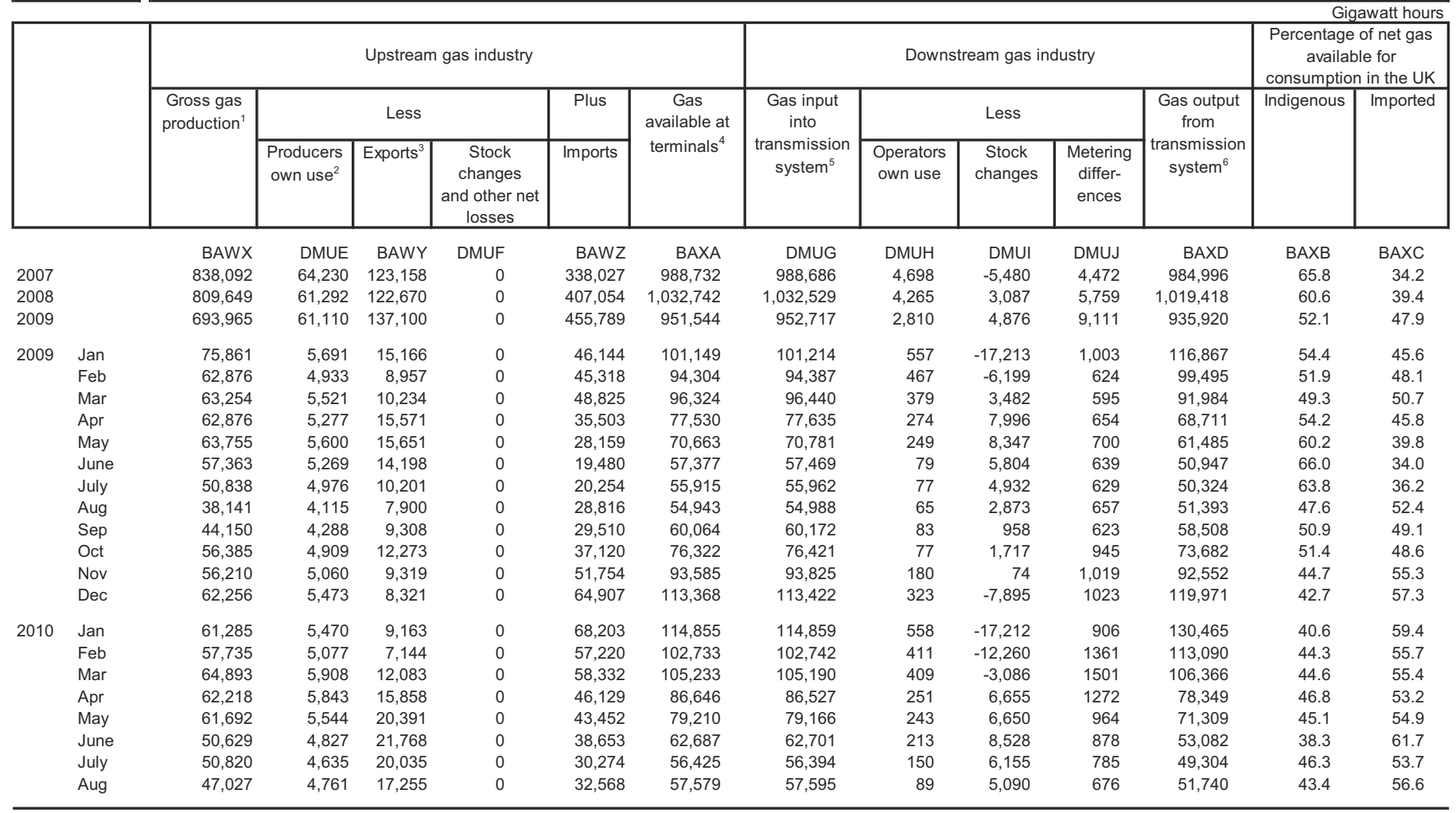

1 Includes waste and own use but excludes gas flared.

2 Gas used for drilling, production and pumping operations.

3 Includes exports direct from the UKCS as well as others carried out by the downstream gas industry from the national transmission system.

4 Gas available at terminals for consumption in the UK as recorded by the terminal operators.

5 Gas input into inland transmission systems. It includes public gas supply, direct supply by North Sea producers, third party supplies, and stock changes. Figures differ from gas available for consumption in the UK mainly because of additional stock changes at local distribution zones. The figures also differ from total consumption (expressed in oil equivalent in Table 6.1) because they exclude producers' and operators' own use and losses.

6 Including public gas supply, direct supplies by North Sea producers, third party supplies and stock changes.

Source: Department of Energy \& Climate Change 03000685056 


\begin{tabular}{|c|c|c|c|c|c|c|c|c|c|c|c|}
\hline & & & & & & & & & & & usand tonne \\
\hline & & & & isumption & & & Total inland & & Stocks at en & f period & \\
\hline & & $\begin{array}{l}\text { Electricity } \\
\text { generators }\end{array}$ & $\begin{array}{c}\text { Cokeovens } \\
\text { and blast } \\
\text { furnaces }\end{array}$ & $\begin{array}{c}\text { Other } \\
\text { conversion } \\
\text { industries } \\
\end{array}$ & Industry & Other $^{3,4,5}$ & consumption & $\begin{array}{c}\text { Electricity } \\
\text { generators }^{6}\end{array}$ & Coke ovens & Other $^{7}$ & $\begin{array}{l}\text { Total } \\
\text { Stocks }\end{array}$ \\
\hline & & BHEC & JW85 & BHEE & BHEF & JW86 & BHEA & JW87 & BHED & JW88 & BHEJ \\
\hline 2007 & & 52,511 & 7,174 & 265 & 1,912 & 1,167 & 63,029 & 11,179 & 1,479 & 1,496 & 14,154 \\
\hline 2008 & & 47,808 & 7,045 & 352 & 1,940 & 1,233 & 58,377 & 14,863 & 1,065 & 1,333 & 17,262 \\
\hline 2009 & & 39,678 & 5,787 & 340 & 1,759 & 1,240 & 48,805 & 21,770 & 806 & 1,514 & 24,090 \\
\hline 2005 & Jan & 5,260 & 466 & 23 & 141 & 94 & 5,983 & 9,114 & 1,145 & 1,423 & 11,682 \\
\hline & Feb & 5,436 & 461 & 16 & 132 & 106 & 6,152 & 7,887 & 1,244 & 1,434 & 10,564 \\
\hline & Mar & 5,595 & 582 & 26 & 147 & 124 & 6,474 & 7,199 & 1,489 & 1,492 & 10,181 \\
\hline & Apr & 4,100 & 490 & 20 & 119 & 73 & 4,803 & 7,216 & 1,525 & 1,416 & 10,157 \\
\hline & May & 3,288 & 502 & 20 & 139 & 88 & 4,038 & 8,378 & 1,527 & 1,337 & 11,242 \\
\hline & June & 3,232 & 620 & 27 & 153 & 99 & 4,132 & 9,840 & 1,651 & 1,347 & 12,837 \\
\hline & July & 2,858 & 531 & 19 & 138 & 83 & 3,628 & 10,924 & 1,760 & 1,177 & 13,862 \\
\hline & Aug & 2,865 & 541 & 22 & 123 & 79 & 3,630 & 12,357 & 1,698 & 1,261 & 15,316 \\
\hline & Sep & 3,154 & 677 & 27 & 191 & 99 & 4,148 & 14,201 & 1,487 & 1,393 & 17,080 \\
\hline & Oct & 3,923 & 534 & 19 & 177 & 79 & 4,732 & 15,387 & 1,340 & 1,444 & 18,171 \\
\hline & Nov & 5,679 & 534 & 17 & 134 & 85 & 6,450 & 15,054 & 1,374 & 1,558 & 17,986 \\
\hline & Dec & 6,669 & 670 & 30 & 187 & 118 & 7,674 & 12,696 & 1,317 & 1,615 & 15,628 \\
\hline 2006 & Jan & 6,602 & 538 & 27 & 118 & 101 & 7,385 & 10,934 & 1,508 & 1,527 & 13,969 \\
\hline & Feb & 5,896 & 513 & 23 & 140 & 102 & 6,674 & 9,844 & 1,611 & 1,556 & 13,010 \\
\hline & Mar & 6,479 & 674 & 28 & 162 & 130 & 7,474 & 9,301 & 1,492 & 1,533 & 12,326 \\
\hline & Apr & 4,090 & 550 & 23 & 142 & 61 & 4,866 & 9,963 & 1,404 & 1,552 & 12,919 \\
\hline & May & 4,112 & 537 & 24 & 149 & 102 & 4,924 & 10,756 & 1,263 & 1,512 & 13,531 \\
\hline & June & 3,514 & 675 & 26 & 165 & 74 & 4,454 & 12,145 & 1,349 & 1,527 & 15,021 \\
\hline & July & 3,886 & 568 & 20 & 149 & 88 & 4,710 & 13,260 & 1,440 & 1,426 & 16,126 \\
\hline & Aug & 3,455 & 542 & 22 & 134 & 81 & 4,233 & 14,389 & 1,198 & 1,378 & 16,965 \\
\hline & Sep & 3,541 & 676 & 31 & 146 & 70 & 4,464 & 15,438 & 1,073 & 1,536 & 18,047 \\
\hline & Oct & 4,078 & 553 & 16 & 128 & 61 & 4,836 & 16,380 & 954 & 1,663 & 18,997 \\
\hline & Nov & 5,820 & 540 & 20 & 135 & 76 & 6,591 & 15,718 & 1,117 & 1,517 & 18,351 \\
\hline & Dec & 5,966 & 683 & 17 & 136 & 111 & 6,912 & 14,813 & 962 & 1,451 & 17,226 \\
\hline 2007 & Jan & 5,905 & 679 & 18 & 151 & 108 & 6,861 & 13,502 & 1,094 & 1,306 & 15,902 \\
\hline & Feb & 4,450 & 554 & 17 & 153 & 99 & 5,273 & 13,329 & 1,294 & 1,376 & 15,998 \\
\hline & Mar & 4,604 & 561 & 14 & 158 & 133 & 5,470 & 13,185 & 1,214 & 1,412 & 15,811 \\
\hline & Apr & 3,203 & 552 & 12 & 155 & 71 & 3,993 & 14,246 & 1,183 & 1,566 & 16,995 \\
\hline & May & 3,792 & 567 & 23 & 160 & 63 & 4,604 & 14,582 & 1,179 & 1,740 & 17,502 \\
\hline & June & 2,942 & 699 & 25 & 160 & 88 & 3,913 & 14,819 & 1,039 & 1,883 & 17,742 \\
\hline & July & 3,571 & 537 & 24 & 157 & 80 & 4,368 & 14,757 & 1,129 & 1,707 & 17,593 \\
\hline & Aug & 3,499 & 557 & 17 & 153 & 84 & 4,310 & 15,215 & 1,207 & 1,783 & 18,205 \\
\hline & Sep & 3,517 & 684 & 25 & 152 & 113 & 4,490 & 15,665 & 1,342 & 1,636 & 18,643 \\
\hline & Oct & 4,833 & 675 & 22 & 154 & 86 & 5,770 & 14,999 & 1,410 & 1,367 & 17,777 \\
\hline & Nov & 5,845 & 436 & 23 & 155 & 99 & 6,558 & 13,060 & 1,464 & 1,441 & 15,964 \\
\hline & Dec & 6,354 & 674 & 26 & 155 & 112 & 7,321 & 11,179 & 1,494 & 1,542 & 14,215 \\
\hline 2008 & Jan & 4,851 & 685 & 25 & 162 & 104 & 5,827 & 10,313 & 1,215 & 1,388 & 12,916 \\
\hline & Feb & 4,338 & 554 & 29 & 154 & 98 & 5,173 & 10,147 & 1,300 & 1,152 & 12,600 \\
\hline & Mar & 4,558 & 533 & 30 & 211 & 107 & 5,440 & 10,073 & 1,033 & 1,349 & 12,456 \\
\hline & Apr & 4,523 & 677 & 27 & 186 & 93 & 5,506 & 9,256 & 1,055 & 1,221 & 11,532 \\
\hline & May & 3,135 & 460 & 24 & 188 & 87 & 3,893 & 10,281 & 1,143 & 1,076 & 12,500 \\
\hline & June & 2,950 & 736 & 29 & 182 & 92 & 3,989 & 11,193 & 1,087 & 1,182 & 13,462 \\
\hline & July & 3,057 & 678 & 19 & 115 & 84 & 3,953 & 12,406 & 1,213 & 1,017 & 14,636 \\
\hline & Aug & 2,488 & 436 & 21 & 122 & 87 & 3,153 & 13,728 & 1,162 & 986 & 15,876 \\
\hline & Sep & 3,370 & 660 & 33 & 118 & 95 & 4,277 & 15,025 & 1,085 & 1,040 & 17,150 \\
\hline & Oct & 4,164 & 663 & 26 & 148 & 98 & 5,099 & 15,468 & 1,058 & 1,042 & 17,568 \\
\hline & Nov & 4,907 & 385 & 26 & 173 & 107 & 5,598 & 15,064 & 1,043 & 1,070 & 17,176 \\
\hline & Dec & 5,461 & 578 & 28 & 113 & 123 & 6,304 & 14,863 & 1,066 & 2,442 & 18,371 \\
\hline 2009 & Jan & 6,062 & 481 & 30 & 132 & 107 & 6,811 & 13,390 & 1,326 & 1,549 & 16,266 \\
\hline & Feb & 5,014 & 418 & 32 & 163 & 109 & 5,735 & 13,490 & 1,548 & 1,193 & 16,231 \\
\hline & Mar & 4,050 & 470 & 32 & 213 & 112 & 4,877 & 13,845 & 1,322 & 1,404 & 16,571 \\
\hline & Apr & 2,746 & 470 & 26 & 168 & 72 & 3,482 & 15,483 & 1,174 & 1,359 & 18,017 \\
\hline & May & 2,538 & 493 & 32 & 87 & 99 & 3,249 & 17,564 & 925 & 1,385 & 19,874 \\
\hline & June & 2,549 & 456 & 29 & 112 & 138 & 3,284 & 18,360 & 870 & 1,765 & 20,995 \\
\hline & July & 2,201 & 478 & 29 & 189 & 68 & 2,966 & 19,769 & 929 & 1,550 & 22,249 \\
\hline & Aug & 1,900 & 479 & 22 & 130 & 93 & 2,624 & 21,702 & 614 & 1,488 & 23,804 \\
\hline & Sep & 2,281 & 490 & 33 & 199 & 105 & 3,108 & 22,863 & 613 & 1,249 & 24,725 \\
\hline & Oct & 3,373 & 503 & 30 & 157 & 103 & 4,167 & 22,513 & 577 & 1,449 & 24,538 \\
\hline & Nov & 3,047 & 513 & 26 & 125 & 109 & 3,820 & 22,296 & 766 & 1,416 & 24,478 \\
\hline & Dec & 3,922 & 535 & 26 & 157 & 125 & 4,766 & 21,770 & 806 & 1,514 & 24,090 \\
\hline 2010 & Jan & 4,914 & 538 & 15 & 146 & 120 & 5,733 & 19,570 & 894 & 1,653 & 22,117 \\
\hline & Feb & 4,280 & 497 & 23 & 143 & 114 & 5,057 & 18,429 & 725 & 1,599 & 20,754 \\
\hline & Mar & 3,253 & 562 & 37 & 138 & 115 & 4,105 & 17,544 & 831 & 1,941 & 20,315 \\
\hline & Apr & 2,632 & 566 & 16 & 137 & 101 & 3,452 & 17,235 & 843 & 1,969 & 20,047 \\
\hline & May & 2,510 & 574 & 19 & 133 & 98 & 3,334 & 16,764 & 879 & 2,136 & 19,780 \\
\hline & June & 2,461 & 519 & 27 & 130 & 95 & 3,232 & 16,728 & 1,189 & 2,243 & 20,161 \\
\hline & July & 2,715 & 524 & 24 & 129 & 91 & 3,483 & 16,403 & 1,064 & 2,156 & 19,623 \\
\hline & Aug & 2,207 & 490 & 24 & 127 & 90 & 2,938 & 16,681 & 1,522 & 1,987 & 20,190 \\
\hline
\end{tabular}

12008 is 4 days longer than the standard 52 week statistical reporting period (SRP) for January to December 2008. This is to enable a smooth transition to publishing data on a calendar month basis from January 2009 rather than 4 and 5 week SRPs used for previous years.

2 Low temperature carbonisation and patent fuel plants.

3 Includes estimates of imports.

4 Includes heat generation (generation of heat for sale under the provision of a contract data are not available before 1999), collieries, domestic, public administration, commerce and agriculture.

5 Heat generation is based on an annual figure and is then split over a 12 monthly period. The 2009 heat generation will not be published until the end of July 2010 . Therefore, the 2008 figure is used as an estimate for 2009.

6 Includes stocks held at ports.

Source: Department of Energy \& Climate Change 03000685056 


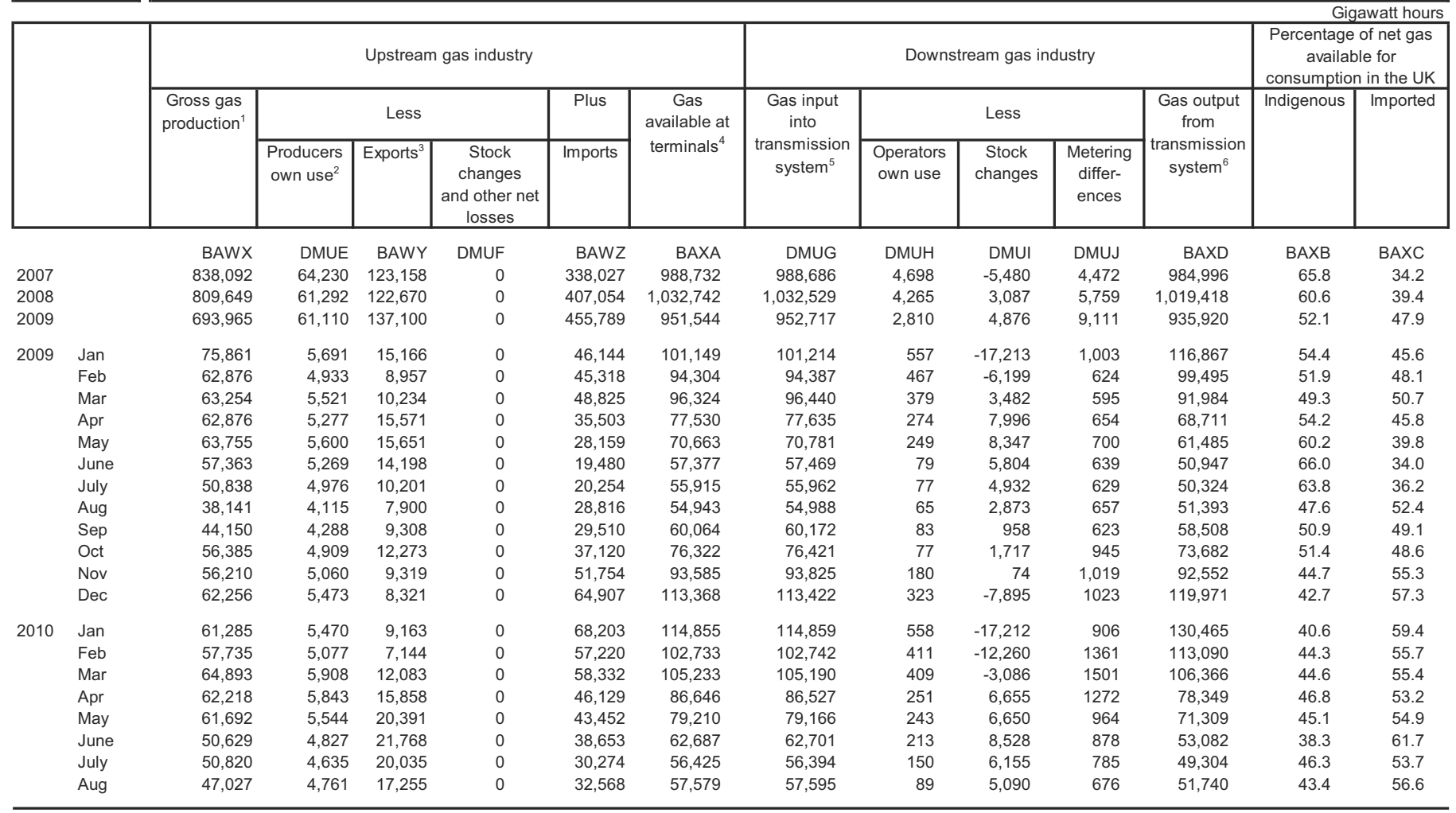

1 Includes waste and own use but excludes gas flared.

2 Gas used for drilling, production and pumping operations.

3 Includes exports direct from the UKCS as well as others carried out by the downstream gas industry from the national transmission system.

4 Gas available at terminals for consumption in the UK as recorded by the terminal operators.

5 Gas input into inland transmission systems. It includes public gas supply, direct supply by North Sea producers, third party supplies, and stock changes. Figures differ from gas available for consumption in the UK mainly because of additional stock changes at local distribution zones. The figures also differ from total consumption (expressed in oil equivalent in Table 6.1) because they exclude producers' and operators' own use and losses.

6 Including public gas supply, direct supplies by North Sea producers, third party supplies and stock changes.

Source: Department of Energy \& Climate Change 03000685056 


\begin{tabular}{|c|c|c|c|c|c|c|c|c|c|c|c|c|c|c|c|c|}
\hline & & \multicolumn{6}{|c|}{ Million tonnes of oil equivalent } & \multicolumn{9}{|c|}{ Terawatt hours } \\
\hline & & \multicolumn{6}{|c|}{ Fuel used } & \multirow{2}{*}{$\begin{array}{l}\text { Electricity } \\
\text { generated }\end{array}$} & \multirow[t]{2}{*}{ 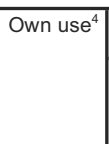 } & \multicolumn{6}{|c|}{ Electricity supplied by type of plant } & \multirow{2}{*}{$\begin{array}{c}\text { Total } \\
\text { electricity } \\
\text { available }\end{array}$} \\
\hline & & $\mathrm{Coal}^{2}$ & $\mathrm{Gas}^{2}$ & \begin{tabular}{c|} 
Nuclear \\
electricity
\end{tabular} & \begin{tabular}{c|} 
Hydro- \\
electricity
\end{tabular} & Wind $^{9}$ & Total $^{3}$ & & & $\begin{array}{c}\text { Conven- } \\
\text { tional } \\
\text { Thermal5 }\end{array}$ & $\begin{array}{c}\text { Combined } \\
\text { Cycle Gas } \\
\text { Turbine }\end{array}$ & Nuclear & Wind $^{9}$ & Other ${ }^{6}$ & Total & \\
\hline & & FTAJ & WSFA & FTAL & FTAM & IWG2 & FTAN & BHJF & BHJJ & FTAB & BAYK & FTAC & IWG3 & FTAD & BHJK & BHJL \\
\hline 2005 & & 31.65 & 25.42 & 18.37 & 0.33 & & 77.48 & 362.21 & 19.97 & 136.00 & 128.18 & 75.17 & & 2.49 & 342.24 & 359.10 \\
\hline 2006 & & 35.00 & 23.92 & 17.13 & 0.32 & & 78.10 & 361.86 & 22.58 & 151.16 & 116.40 & 69.24 & & 2.64 & 339.28 & 356.91 \\
\hline 2007 & & 31.99 & 27.50 & 14.04 & 0.36 & 0.31 & 75.57 & 361.41 & 21.17 & 138.88 & 137.66 & 57.25 & 3.57 & 2.15 & 340.24 & 358.08 \\
\hline 2008 & & 29.00 & 29.62 & 11.91 & 0.37 & 0.46 & 73.26 & 355.28 & 20.04 & 121.88 & 157.42 & 47.67 & 5.36 & 2.39 & 335.24 & 359.81 \\
\hline $2009^{p}$ & & 23.82 & 28.01 & 15.23 & 0.37 & 0.59 & 69.79 & 341.47 & 19.58 & 101.19 & 147.92 & 62.76 & 6.90 & 2.46 & 321.89 & 340.51 \\
\hline \multirow[t]{6}{*}{2007} & Jul & 2.18 & 2.01 & 1.36 & 0.02 & 0.02 & 5.68 & 27.10 & 1.72 & 9.44 & 10.01 & 5.55 & 0.25 & 0.13 & 25.38 & 26.84 \\
\hline & Aug & 2.13 & 2.07 & 1.22 & 0.02 & 0.02 & 5.56 & 26.60 & 1.68 & 9.29 & 10.23 & 4.99 & 0.29 & 0.14 & 24.92 & 26.65 \\
\hline & Sep & 2.13 & 2.10 & 1.27 & 0.02 & 0.03 & 5.64 & 27.05 & 1.62 & 8.91 & 10.86 & 5.19 & 0.33 & 0.10 & 25.43 & 26.92 \\
\hline & Oct & 2.95 & 2.31 & 1.10 & 0.02 & 0.02 & 6.55 & 31.04 & 1.87 & 12.49 & 11.81 & 4.49 & 0.24 & 0.19 & 29.17 & 30.33 \\
\hline & Nov & 3.58 & 2.17 & 1.11 & 0.03 & 0.03 & 7.10 & 33.46 & 2.01 & 15.57 & 10.74 & 4.54 & 0.35 & 0.19 & 31.45 & 32.67 \\
\hline & Dec & 3.88 & 2.18 & 1.14 & 0.04 & 0.04 & 7.43 & 34.92 & 2.12 & 16.75 & 10.63 & 4.63 & 0.43 & 0.21 & 32.81 & 33.58 \\
\hline \multirow[t]{12}{*}{2008} & Jan & 2.96 & 2.59 & 1.15 & 0.05 & 0.05 & 6.94 & 34.32 & 1.85 & 12.10 & 14.69 & 4.61 & 0.60 & 0.21 & 32.47 & 34.38 \\
\hline & Feb & 2.64 & 2.63 & 1.12 & 0.05 & 0.04 & 6.61 & 31.97 & 1.70 & 10.78 & 14.08 & 4.48 & 0.49 & 0.22 & 30.27 & 31.98 \\
\hline & Mar & 2.76 & 2.71 & 1.01 & 0.05 & 0.05 & 6.70 & 32.54 & 1.70 & 11.32 & 14.46 & 4.05 & 0.57 & 0.19 & 30.84 & 32.44 \\
\hline & Apr & 2.76 & 2.43 & 0.80 & 0.03 & 0.03 & 6.19 & 29.98 & 1.59 & 11.75 & 12.78 & 3.21 & 0.36 & 0.21 & 28.39 & 30.20 \\
\hline & May & 1.89 & 2.44 & 0.99 & 0.02 & 0.02 & 5.47 & 26.59 & 1.49 & 8.05 & 12.76 & 3.94 & 0.27 & 0.18 & 25.10 & 27.31 \\
\hline & Jun & 1.75 & 2.34 & 0.98 & 0.01 & 0.03 & 5.24 & 25.46 & 1.46 & 7.50 & 12.26 & 3.92 & 0.32 & 0.18 & 24.00 & 26.05 \\
\hline & Jul & 1.84 & 2.48 & 0.90 & 0.01 & 0.03 & 5.39 & 26.47 & 1.54 & 7.83 & 13.15 & 3.60 & 0.35 & 0.18 & 24.93 & 27.13 \\
\hline & Aug & 1.48 & 2.62 & 0.97 & 0.02 & 0.03 & 5.25 & 26.16 & 1.47 & 6.49 & 13.89 & 3.90 & 0.33 & 0.17 & 24.69 & 26.78 \\
\hline & Sep & 2.02 & 2.37 & 0.87 & 0.02 & 0.03 & 5.49 & 26.57 & 1.58 & 8.95 & 12.15 & 3.47 & 0.31 & 0.18 & 25.00 & 26.95 \\
\hline & Oct & 2.53 & 2.45 & 0.96 & 0.04 & 0.06 & 6.23 & 30.19 & 1.75 & 11.14 & 12.50 & 3.85 & 0.64 & 0.22 & 28.44 & 29.74 \\
\hline & Nov & 3.00 & 2.26 & 0.96 & 0.04 & 0.05 & 6.54 & 31.18 & 1.83 & 12.88 & 11.69 & 3.85 & 0.60 & 0.20 & 29.34 & 30.65 \\
\hline & Dec & 3.32 & 2.25 & 1.20 & 0.04 & 0.04 & 7.09 & 33.82 & 2.05 & 14.29 & 11.83 & 4.79 & 0.53 & 0.24 & 31.77 & 32.78 \\
\hline \multirow[t]{12}{*}{2009} & Jan & 3.59 & 2.21 & 0.98 & 0.04 & 0.07 & 7.14 & 34.72 & 2.05 & 15.93 & 11.52 & 4.06 & 0.80 & 0.25 & 32.66 & 34.19 \\
\hline & Feb & 3.03 & 1.91 & 1.27 & 0.03 & 0.04 & 6.48 & 30.59 & 1.89 & 12.83 & 9.93 & 5.22 & 0.50 & 0.24 & 28.70 & 30.17 \\
\hline & Mar & 2.45 & 2.21 & 1.49 & 0.04 & 0.06 & 6.42 & 30.79 & 1.83 & 10.20 & 11.52 & 6.14 & 0.70 & 0.26 & 28.96 & 30.54 \\
\hline & Apr & 1.66 & 2.16 & 1.42 & 0.03 & 0.04 & 5.43 & 26.07 & 1.47 & 6.74 & 11.32 & 5.85 & 0.44 & 0.19 & 24.60 & 26.64 \\
\hline & May & 1.53 & 2.27 & 1.19 & 0.02 & 0.06 & 5.19 & 25.47 & 1.45 & 6.35 & 11.90 & 4.91 & 0.66 & 0.19 & 24.01 & 26.39 \\
\hline & June & 1.52 & 2.05 & 1.38 & 0.01 & 0.03 & 5.11 & 24.88 & 1.51 & 6.39 & 10.89 & 5.68 & 0.37 & 0.18 & 23.37 & 25.21 \\
\hline & July & 1.34 & 2.33 & 1.39 & 0.01 & 0.03 & 5.23 & 26.23 & 1.52 & 5.59 & 12.93 & 5.72 & 0.40 & 0.19 & 24.71 & 26.72 \\
\hline & Aug & 1.15 & 2.41 & 1.34 & 0.03 & 0.05 & 5.09 & 25.55 & 1.44 & 4.96 & 12.77 & 5.53 & 0.58 & 0.18 & 24.11 & 25.78 \\
\hline & Sep & 1.37 & 2.49 & 1.18 & 0.04 & 0.05 & 5.24 & 26.00 & 1.47 & 5.86 & 13.01 & 4.85 & 0.57 & 0.13 & 24.54 & 25.66 \\
\hline & Oct & 2.03 & 2.56 & 1.10 & 0.03 & 0.05 & 5.91 & 28.96 & 1.57 & 8.58 & 13.47 & 4.52 & 0.54 & 0.18 & 27.38 & 28.06 \\
\hline & Nov & 1.81 & 2.65 & 1.23 & 0.05 & 0.07 & 5.96 & 29.83 & 1.59 & 7.86 & 14.06 & 5.05 & 0.77 & 0.24 & 28.24 & 29.34 \\
\hline & Dec & 2.34 & 2.76 & 1.27 & 0.03 & 0.05 & 6.59 & 32.38 & 1.77 & 9.92 & 14.59 & 5.22 & 0.58 & 0.24 & 30.60 & 31.81 \\
\hline \multirow[t]{8}{*}{2010} & Jan & 2.98 & 2.61 & 1.40 & 0.02 & 0.06 & 7.25 & 35.13 & 1.98 & 12.66 & 13.94 & 5.77 & 0.67 & 0.31 & 33.15 & 33.91 \\
\hline & Feb & 2.59 & 2.34 & 1.21 & 0.01 & 0.04 & 6.33 & 30.72 & 1.71 & 10.94 & 12.60 & 4.99 & 0.41 & 0.29 & 29.01 & 29.76 \\
\hline & Mar & 1.94 & 2.68 & 1.40 & 0.02 & 0.06 & 6.25 & 31.06 & 1.65 & 8.27 & 14.54 & 5.76 & 0.69 & 0.33 & 29.40 & 30.31 \\
\hline & Apr & 1.57 & 2.48 & 1.13 & 0.02 & 0.04 & 5.35 & 26.41 & 1.41 & 6.59 & 13.08 & 4.66 & 0.50 & 0.25 & 25.00 & 26.29 \\
\hline & May & 1.49 & 2.51 & 0.98 & 0.01 & 0.03 & 5.13 & 25.40 & 1.35 & 6.22 & 13.40 & 4.03 & 0.37 & 0.25 & 24.06 & 25.68 \\
\hline & June & 1.47 & 2.23 & 0.95 & 0.01 & 0.03 & 4.79 & 23.47 & 1.28 & 6.14 & 11.81 & 3.91 & 0.34 & 0.23 & 22.19 & 24.24 \\
\hline & July & 1.64 & 2.12 & 0.97 & 0.02 & 0.06 & 4.92 & 23.99 & 1.30 & 6.76 & 11.15 & 3.98 & 0.69 & 0.23 & 22.69 & 25.03 \\
\hline & Aug & 1.32 & 2.20 & 0.99 & 0.02 & 0.05 & 4.71 & 23.30 & 1.25 & 5.58 & 11.67 & 4.06 & 0.63 & 0.24 & 22.05 & 24.59 \\
\hline
\end{tabular}

1 Fuel used and electricity generated by major power producers (National Power, PowerGen, Nuclear Electric, National Grid Company, Scottish Power,

Hydro Electric, Scottish Nuclear, NIGEN, Coolkeeragh Power Ltd, Balyumford Power Ltd, Midlands Electricity, South Western Electricity, Teeside Power Ltd, Lakeland Power Ltd, Fibropower Ltd, Corby Power Ltd, Peterborough Power Ltd, Fibrogen Ltd, and Regional Power Ltd) and electricity available through the

grid in England and Wales and from distribution companies in Scotland and Northern Ireland.

2 Includes quantities used in the production of steam for sale.

3 Including oil used in gas turbine and diesel plant and for lighting up coal-fired boilers and orimulsion.

4 Including refuse-derived fuel, natural gas and sour gas.

5 Used in works and for pumping at pumped storage stations.

6 Coal Oil (including Orimulsion) and mixed or dual-fired (including gas)

7 Including gas turbine, diesel, wind and hydro-electric plant.

8 Including net imports and purchases from outside sources, mainly UKAEA and British Nuclear Fuels Plc and net of supplies direct from generators to final consumers.

9 From September 2008 (with data from January 2007), major power producers also include major wind farm companies (Airtricity; Cumbria Wind Farms; Fred Olsen; HG Capital; Kentish Flats; RES Generation Ltd). Generation from wind by major power producers listed in note 1 above is also included from these dates. 


\begin{tabular}{|c|c|c|c|c|c|c|c|c|c|c|c|c|}
\hline \multicolumn{2}{|c|}{ Table 6.7} & \multicolumn{11}{|c|}{ Sales by the gas and public electricity supply systems } \\
\hline & & \multicolumn{7}{|c|}{ Gas: Gigawatt hours } & \multicolumn{4}{|c|}{ Electricity: Terawatt hours } \\
\hline & & $\begin{array}{c}\text { Electricity } \\
\text { generators }\end{array}$ & $\begin{array}{c}\text { Heat } \\
\text { generation }\end{array}$ & $\begin{array}{l}\text { Iron and } \\
\text { steel } \\
\text { industry }\end{array}$ & $\begin{array}{c}\text { Other } \\
\text { industries }\end{array}$ & Domestic & Other $^{2}$ & Total & Industrial $^{3}$ & Domestic & Other ${ }^{4}$ & Total \\
\hline & & BBKF & WSFM & BBKG & $\mathrm{BBKH}$ & BBKI & BBKJ & BBKK & FTAE & FTAG & FTAH & FTAI \\
\hline 1996 & & 199,892 & & 21,660 & 160,992 & 375,841 & 119,336 & 877,721 & 97.99 & 107.52 & 93.37 & 298.87 \\
\hline 1997 & & 250,154 & & 20,934 & 167,080 & 345,532 & 113,668 & 897,368 & 98.56 & 104.45 & 97.76 & 300.75 \\
\hline 2002 & & 329,442 & 22,010 & 8,791 & 156,285 & 376,372 & 100,833 & 993,733 & 101.59 & 114.52 & 103.68 & 319.80 \\
\hline 2003 & & 323,926 & 19,830 & 10,326 & 155,813 & 386,486 & 106,737 & $1,003,119$ & 103.33 & 115.76 & 105.23 & 324.33 \\
\hline 2004 & & 341,111 & 19,886 & 9,715 & 143,959 & 396,411 & 112,065 & $1,023,147$ & 103.24 & 115.53 & 104.94 & 323.71 \\
\hline 2005 & & 333,834 & 20,671 & 8,410 & 140,328 & 381,879 & 106,653 & 991,775 & 105.88 & 116.81 & 106.38 & 329.07 \\
\hline 2006 & & 308,911 & 22,023 & 8,406 & 137,280 & 365,850 & 103,174 & 945,644 & 105.43 & 116.45 & 106.42 & 328.30 \\
\hline 2007 & & 355,292 & 23,640 & 7,323 & 125,988 & 352,868 & 94,826 & 959,937 & 105.76 & 122.76 & 102.73 & 331.25 \\
\hline 2008 & & 376,380 & 23,382 & 6,920 & 131,766 & 359,554 & 95,635 & 995,637 & 102.54 & 125.81 & 103.56 & 331.91 \\
\hline 2009 & & 355,730 & 24,716 & 5,037 & 109,384 & 334,823 & 83,099 & 912,789 & 92.23 & 122.54 & 99.04 & 313.82 \\
\hline \multirow[t]{4}{*}{2003} & Q1 & 79,391 & 6,582 & 2,678 & 50,535 & 157,739 & 35,445 & 332,370 & 26.72 & 34.48 & 27.00 & 88.20 \\
\hline & Q2 & 76,333 & 4,055 & 2,597 & 36,710 & 62,659 & 22,532 & 204,885 & 25.88 & 25.00 & 25.31 & 76.19 \\
\hline & Q3 & 82,069 & 3,300 & 2,339 & 27,391 & 34,288 & 12,661 & 162,047 & 25.31 & 22.81 & 24.74 & 72.87 \\
\hline & Q4 & 86,133 & 5,893 & 2,713 & 41,178 & 131,802 & 36,099 & 303,816 & 25.42 & 33.47 & 28.18 & 87.07 \\
\hline \multirow[t]{4}{*}{2004} & Q1 & 83,687 & 6,740 & 2,573 & 49,993 & 159,663 & 41,113 & 343,768 & 27.86 & 35.18 & 27.62 & 90.66 \\
\hline & Q2 & 81,845 & 4,780 & 2,558 & 31,460 & 66,867 & 24,098 & 211,609 & 24.52 & 23.81 & 24.16 & 72.49 \\
\hline & Q3 & 85,612 & 4,157 & 2,248 & 23,768 & 37,341 & 15,189 & 168,315 & 25.73 & 22.42 & 25.35 & 73.51 \\
\hline & Q4 & 89,086 & 6,167 & 2,336 & 38,952 & 132,540 & 33,075 & 302,156 & 25.13 & 34.11 & 27.82 & 87.06 \\
\hline \multirow[t]{4}{*}{2005} & Q1 & 78,103 & 6,972 & 2,391 & 48,309 & 156,131 & 37,682 & 329,588 & 27.11 & 33.57 & 28.28 & 88.97 \\
\hline & Q2 & 84,877 & 5,012 & 2,306 & 31,687 & 68,383 & 24,099 & 216,365 & 26.67 & 25.97 & 25.22 & 77.87 \\
\hline & Q3 & 88,146 & 4,222 & 1,783 & 24,106 & 33,037 & 13,860 & 165,154 & 24.94 & 24.00 & 24.57 & 73.51 \\
\hline & Q4 & 77,245 & 6,282 & 1,989 & 38,765 & 126,458 & 31,850 & 282,589 & 27.16 & 33.27 & 28.30 & 88.73 \\
\hline \multirow[t]{4}{*}{2006} & Q1 & 66,793 & 7,034 & 2,162 & 55,807 & 157,999 & 40,365 & 330,160 & 28.03 & 34.99 & 28.75 & 91.77 \\
\hline & Q2 & 73,941 & 4,945 & 2,232 & 26,462 & 67,051 & 22,152 & 196,782 & 25.32 & 25.66 & 25.14 & 76.12 \\
\hline & Q3 & 79,881 & 4,058 & 2,028 & 19,934 & 28,698 & 12,914 & 147,512 & 25.82 & 23.34 & 25.03 & 74.19 \\
\hline & Q4 & 88,295 & 5,986 & 1,985 & 35,078 & 112,102 & 27,743 & 271,190 & 26.25 & 32.46 & 27.50 & 86.21 \\
\hline \multirow[t]{4}{*}{2007} & Q1 & 92,611 & 6,641 & 1,958 & 42,057 & 137,102 & 36,101 & 316,470 & 26.55 & 33.03 & 28.46 & 88.04 \\
\hline & Q2 & 93,718 & 4,698 & 1,814 & 25,487 & 56,001 & 18,790 & 200,509 & 26.03 & 24.91 & 25.17 & 76.11 \\
\hline & Q3 & 79,670 & 4,165 & 1,698 & 23,699 & 33,990 & 16,490 & 159,712 & 25.84 & 23.72 & 24.83 & 74.40 \\
\hline & Q4 & 86,152 & 6,405 & 1,841 & 36,666 & 125,848 & 28,349 & 285,261 & 27.33 & 33.39 & 27.77 & 88.50 \\
\hline \multirow[t]{4}{*}{2008} & Q1 & 100,908 & 7,027 & 1,903 & 39,921 & 144,299 & 40,811 & 334,869 & 26.55 & 35.05 & 29.40 & 90.99 \\
\hline & Q2 & 91,524 & 5,127 & 1,956 & 28,170 & 61,747 & 21,546 & 210,070 & 25.46 & 25.60 & 25.36 & 76.42 \\
\hline & Q3 & 93,861 & 4,405 & 1,668 & 23,454 & 31,265 & 14,853 & 169,505 & 25.05 & 24.07 & 24.69 & 73.82 \\
\hline & Q4 & 87,287 & 6,603 & 1,291 & 34,104 & 126,004 & 28,263 & 283,552 & 25.88 & 33.12 & 27.71 & 86.70 \\
\hline \multirow[t]{4}{*}{2009} & Q1 & 81,781 & 7,526 & 1,200 & 33,784 & 146,022 & 31,657 & 301,970 & 23.97 & 36.26 & 27.24 & 87.46 \\
\hline & Q2 & 82,778 & 5,377 & 1,137 & 18,815 & 52,958 & 15,597 & 176,661 & 21.90 & 26.89 & 22.99 & 71.77 \\
\hline & Q3 & 91,757 & 4,776 & 1,293 & 18,423 & 28,763 & 9,928 & 154,941 & 21.55 & 25.77 & 23.99 & 71.32 \\
\hline & Q4 & 99,415 & 7,037 & 1,407 & 38,362 & 107,079 & 25,917 & 279,217 & 24.82 & 33.63 & 24.82 & 83.27 \\
\hline \multirow[t]{2}{*}{2010} & Q1 & 97,094 & 7,526 & 1,424 & 41,767 & 158,732 & 37,107 & 343,651 & 23.35 & 36.44 & 26.31 & 86.10 \\
\hline & Q2 & 90,607 & 5,377 & 1,458 & 21,443 & 58,120 & 17,093 & 194,098 & 21.97 & 25.69 & 21.59 & 69.26 \\
\hline
\end{tabular}

1 Power stations belonging to major generating companies, industrial establishments and transport undertakings generating 1 gigawatt or more a year.

2 Public administration, commerce and agriculture.

3 Manufacturing industry, construction, energy and water supply industries.

4 Commercial premises, public administration, transport and agriculture.

Source: Department of Energy \& Climate Change 03000685056 


\section{Table 6.8}

Indigenous production, refinery receipts, arrivals and shipments of oil ${ }^{1}$

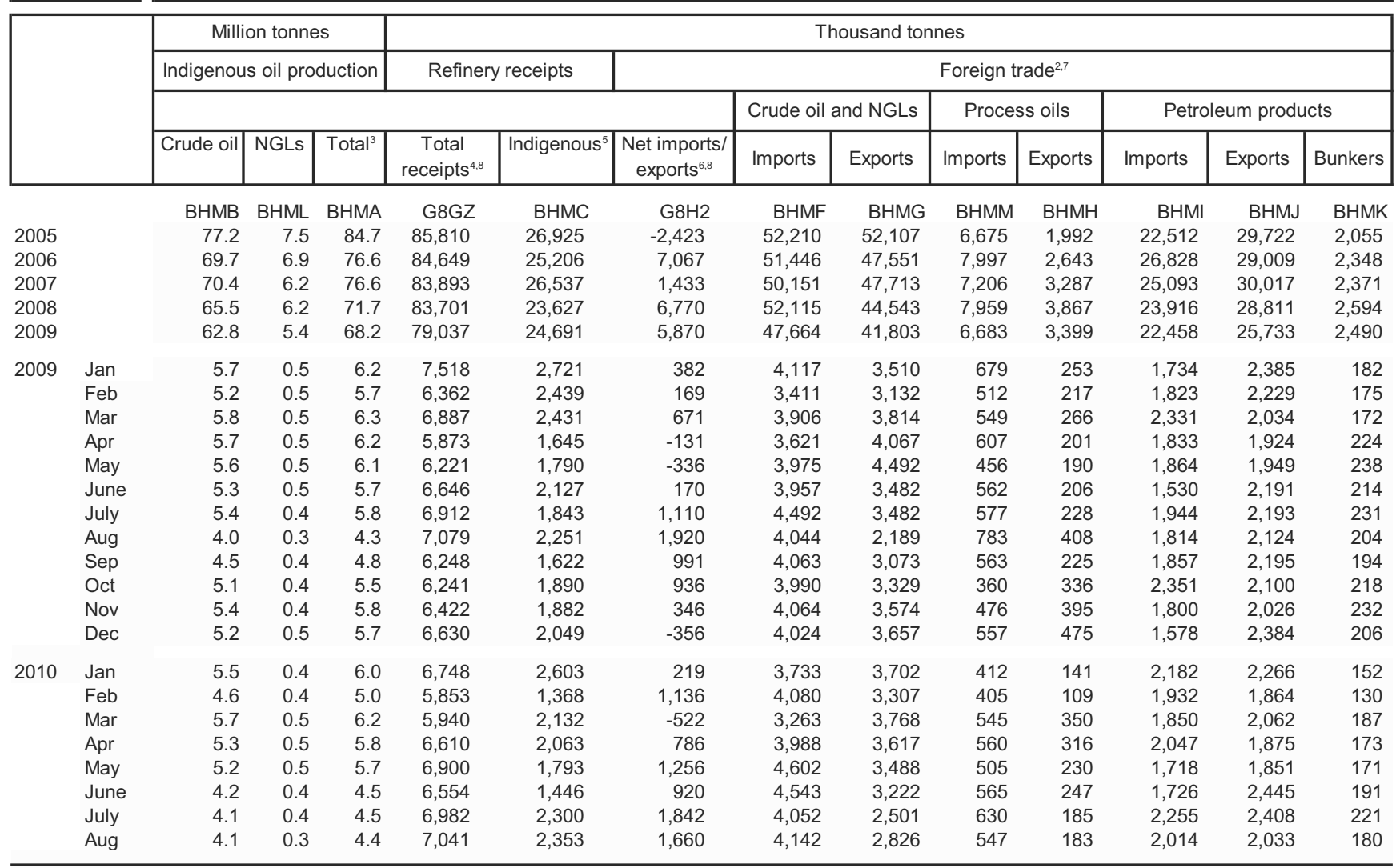

1 The term indigenous is used in this table for convenience to include oil from the UK Continental Shelf as well as the small amounts produced on the mainland.

2 Foreign trade as recorded by the petroleum industry and may differ from figures published in the Overseas Trade Statistics .

3 Crude oil plus condensates and petroleum gases derived at onshore treatment plants.

4 Crude oil, natural gas liquids (NGLs) and process oils (i.e.partly refined oils).

5 Crude oils plus NGLs.

6 Net imports (+) or net exports (-) of oil and oil products.

7 From January 1993 arrivals of petroleum industry and marine bunkers contain estimated additions to allow for (temporarily) missing imports data.

8 There have been some modest changes made to this table following the review announced by DTI in the June 2006 edition of Energy Trends. The review was aimed to improve data coherency and coverage between the different quarterly and monthly DTI tables.

Sources: Department of Energy and Climate Change 03000685056 


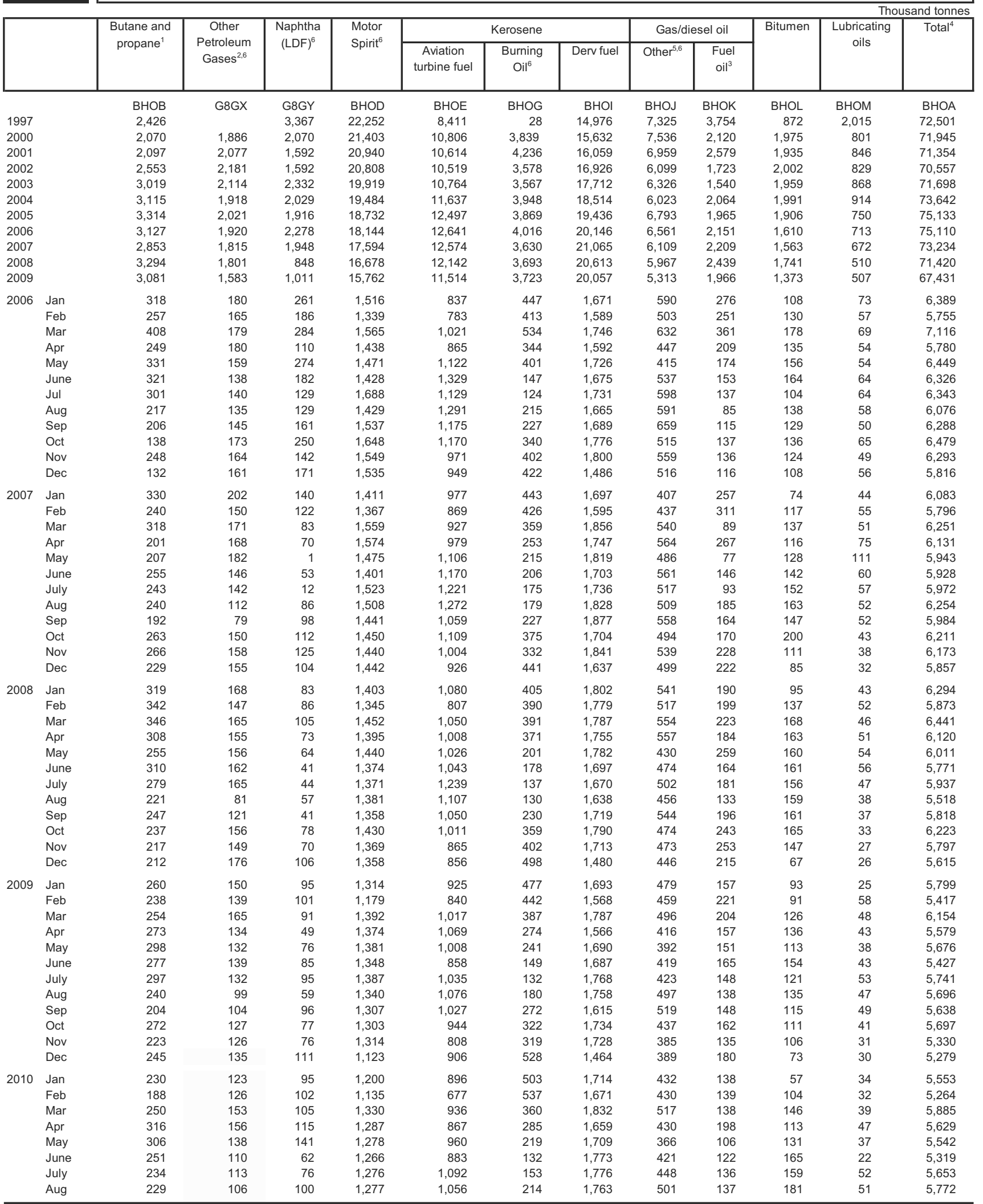

1 Including amounts for petro-chemicals.

2 Ethane and other petroleum gases (OPG)

3 Excluding Orimulsion and refinery fuel

4 Including other petroleum gases, aviation spirit, wide-cut gasoline, industrial and white spirits, petroleum wax, non-domestic standard burning oil and miscellaneous

products, but excluding refinery fuel.

5 Includes gas, oil, marine diesel oil and middle distillate feedstock.

6 There have been some modest changes made to this table following the review announced by DTI in the June 2006 edition of Energy Trends. The review was aimed to improve data coherency and coverage between the different quarterly and monthly tables. Other petroleum gases are now shown. Middle distillate feedstock is now included with other gas/diesel oil rather than previously being combined with naphtha. Unleaded motor spirit and premier burning oil have been discontinued.

Source: Department of Energy \& Climate Change 03000685056 


\begin{tabular}{|c|c|c|c|c|c|c|c|c|c|c|c|c|}
\hline \multicolumn{2}{|c|}{ Table 6.10} & \multicolumn{11}{|c|}{ Motor vehicles:new registrations in Great Britain } \\
\hline & & & & & & & & & & & & Thousands \\
\hline & & \multirow{2}{*}{$\begin{array}{l}\text { Private and } \\
\text { light goods } \\
\text { (PLG) }\end{array}$} & \multicolumn{2}{|c|}{ of which: } & \multirow[t]{2}{*}{ Motorcycles } & \multirow{2}{*}{$\begin{array}{l}\text { Other goods } \\
\text { vehicles }\end{array}$} & \multirow{2}{*}{$\begin{array}{c}\text { Public } \\
\text { transport } \\
\text { vehicles }\end{array}$} & \multirow{2}{*}{$\begin{array}{c}\text { Agriculture } \\
\text { machines }\end{array}$} & \multirow{2}{*}{$\begin{array}{c}\text { Other } \\
\text { licensed } \\
\text { vehicles }\end{array}$} & \multirow{2}{*}{\begin{tabular}{|c|} 
Vehicles \\
exempt from \\
tax
\end{tabular}} & \multirow[t]{2}{*}{ All vehicles } & \multirow{2}{*}{$\begin{array}{c}\text { Of which } \\
\text { bodytype } \\
\text { cars }\end{array}$} \\
\hline & & & $\begin{array}{c}\text { PLG: } \\
\text { Bodytype } \\
\text { cars }\end{array}$ & $\begin{array}{l}\text { PLG: Others } \\
\text { (mainly } \\
\text { light goods) }\end{array}$ & & & & & & & & \\
\hline & & BMAK & BMAA & BMAE & BMAL & BBJY & BBJZ & BBKA & BBKB & BBKC & BBKD & BBKE \\
\hline 2005 & & $1,959.1$ & $1,765.5$ & 193.5 & 111.5 & 27.0 & 7.2 & 27.6 & 5.5 & 233.3 & $2,371.2$ & $1,968.3$ \\
\hline 2006 & & $2,539.1$ & $2,191.4$ & 348.0 & 143.0 & 41.2 & 9.1 & 26.7 & 10.5 & 227.4 & $2,996.9$ & $2,390.2$ \\
\hline 2007 & & $2,188.3$ & $1,891.9$ & 296.4 & 138.4 & 47.0 & 8.3 & 29.9 & 9.6 & 250.7 & $2,672.2$ & $2,112.0$ \\
\hline 2008 & & $2,499.1$ & $2,241.7$ & 338.4 & 131.9 & 47.9 & 7.6 & 24.0 & 9.4 & 193.7 & $2,913.6$ & $2,340.0$ \\
\hline 2009 & & $2,603.7$ & $2,266.2$ & 337.0 & 132.1 & 51.3 & 8.9 & 23.5 & 9.2 & 193.2 & $3,021.4$ & $2,443.5$ \\
\hline \multirow[t]{12}{*}{2008} & Jan & 165.6 & 143.2 & 22.3 & 6.2 & 3.9 & 0.6 & 1.5 & 1.0 & 17.4 & 196.1 & 158.9 \\
\hline & Feb & 73.2 & 58.7 & 14.4 & 5.5 & 2.9 & 0.5 & 1.3 & 0.9 & 11.2 & 95.4 & 67.8 \\
\hline & Mar & 470.4 & 416.9 & 53.5 & 19.0 & 5.3 & 1.0 & 4.2 & 1.1 & 34.5 & 535.6 & 449.0 \\
\hline & Apr & 180.7 & 154.1 & 26.6 & 13.2 & 4.2 & 0.7 & 3.4 & 1.1 & 21.7 & 224.9 & 173.4 \\
\hline & May & 184.5 & 158.0 & 26.6 & 13.8 & 4.0 & 0.8 & 3.1 & 1.0 & 21.4 & 228.6 & 176.5 \\
\hline & Jun & 215.9 & 186.5 & 29.4 & 14.8 & 4.2 & 0.8 & 3.0 & 0.9 & 23.0 & 262.6 & 206.6 \\
\hline & Jul & 155.3 & 132.5 & 22.7 & 14.1 & 3.6 & 0.7 & 3.6 & 0.8 & 21.4 & 199.5 & 151.1 \\
\hline & Aug & 62.5 & 50.7 & 11.9 & 10.1 & 2.8 & 0.5 & 2.3 & 0.6 & 14.5 & 93.2 & 62.5 \\
\hline & Sep & 338.5 & 300.8 & 37.6 & 16.3 & 4.9 & 1.2 & 2.8 & 0.7 & 30.3 & 394.6 & 328.6 \\
\hline & Oct & 127.7 & 109.0 & 18.7 & 11.3 & 4.0 & 0.7 & 1.9 & 0.6 & 21.3 & 167.6 & 127.5 \\
\hline & Nov & 101.1 & 85.4 & 15.7 & 7.6 & 3.9 & 0.5 & 1.5 & 0.6 & 18.1 & 133.3 & 101.2 \\
\hline & Dec & 112.9 & 96.0 & 16.9 & 6.7 & 3.4 & 0.5 & 1.2 & 0.4 & 15.8 & 140.8 & 109.5 \\
\hline \multirow[t]{12}{*}{2009} & Jan & 108.6 & 96.9 & 11.6 & 6.1 & 2.5 & 0.6 & 1.6 & 0.5 & 14.4 & 134.2 & 108.8 \\
\hline & Feb & 50.7 & 43.5 & 7.2 & 4.0 & 1.7 & 0.4 & 1.3 & 0.4 & 11.1 & 69.5 & 52.8 \\
\hline & Mar & 314.4 & 283.8 & 30.7 & 16.7 & 3.2 & 1.0 & 4.7 & 0.6 & 29.5 & 370.1 & 310.7 \\
\hline & Apr & 130.5 & 116.0 & 14.5 & 11.9 & 2.4 & 0.7 & 3.3 & 0.5 & 18.6 & 168.0 & 132.0 \\
\hline & May & 131.5 & 117.8 & 13.6 & 11.6 & 2.1 & 0.6 & 2.6 & 0.4 & 17.4 & 166.3 & 132.9 \\
\hline & Jun & 173.6 & 155.7 & 18.0 & 11.6 & 2.1 & 0.6 & 3.0 & 0.5 & 20.7 & 212.1 & 173.1 \\
\hline & Jul & 151.4 & 136.7 & 14.7 & 10.6 & 1.9 & 0.6 & 3.1 & 0.5 & 20.9 & 189.0 & 154.7 \\
\hline & Aug & 62.5 & 54.4 & 8.2 & 7.1 & 1.5 & 0.4 & 1.8 & 0.4 & 13.7 & 87.5 & 65.3 \\
\hline & Sep & 368.2 & 338.6 & 29.6 & 13.0 & 5.4 & 0.9 & 2.3 & 0.5 & 29.6 & 420.0 & 364.9 \\
\hline & Oct & 162.7 & 147.4 & 15.3 & 8.4 & 1.0 & 0.6 & 1.5 & 0.4 & 21.3 & 195.9 & 166.5 \\
\hline & Nov & 155.1 & 139.3 & 15.8 & 5.8 & 1.5 & 0.5 & 1.4 & 0.4 & 19.6 & 184.3 & 156.5 \\
\hline & Dec & 149.8 & 135.4 & 14.4 & 4.7 & 1.6 & 0.4 & 1.1 & 0.3 & 16.4 & 174.4 & 150.1 \\
\hline \multirow[t]{8}{*}{2010} & Jan & 137.4 & 125.8 & 11.7 & 3.4 & 1.1 & 0.4 & 1.2 & 0.5 & 14.5 & 158.5 & 138.9 \\
\hline & Feb & 64.6 & 55.5 & 9.1 & 3.3 & 1.1 & 0.3 & 1.1 & 0.4 & 11.6 & 82.3 & 65.2 \\
\hline & Mar & 398.0 & 362.3 & 35.6 & 14.9 & 3.0 & 1.2 & 4.2 & 0.6 & 33.6 & 455.5 & 393.4 \\
\hline & Apr & 142.2 & 126.7 & 15.5 & 10.0 & 1.9 & 0.6 & 2.9 & 0.5 & 21.0 & 179.1 & 145.3 \\
\hline & May & 150.5 & 132.5 & 18.0 & 10.4 & 1.9 & 0.6 & 2.4 & 0.5 & 20.5 & 186.8 & 150.6 \\
\hline & Jun & 190.1 & 169.3 & 20.8 & 10.6 & 2.3 & 0.6 & 2.6 & 0.5 & 24.4 & 231.0 & 190.8 \\
\hline & Jul & 130.9 & 112.9 & 18.0 & 10.0 & 2.0 & 0.4 & 2.8 & 0.6 & 24.1 & 170.7 & 133.6 \\
\hline & Aug & 50.6 & 41.7 & 9.0 & 6.3 & 1.6 & 0.3 & 1.7 & 0.5 & 14.9 & 76.0 & 53.7 \\
\hline
\end{tabular}

Source: Department for Transport

\begin{tabular}{|c|c|c|c|c|c|c|c|c|c|c|}
\hline \multicolumn{2}{|c|}{ Table 6.11} & \multicolumn{9}{|c|}{ Road casualties in Great Britain } \\
\hline & & \multirow{2}{*}{\multicolumn{3}{|c|}{ Total casualties }} & \multirow{2}{*}{\multicolumn{3}{|c|}{ Severity }} & & & Number \\
\hline & & & & & & & & \multicolumn{3}{|c|}{ All severities } \\
\hline & & All ages & Under 16 years & Killed & $\begin{array}{l}\text { Seriously } \\
\text { injured }\end{array}$ & $\begin{array}{l}\text { Slightly } \\
\text { injured }\end{array}$ & Pedestrians & Pedal cyclists & $\begin{array}{l}\text { Motorcyclists } \\
\text { and their } \\
\text { passengers }\end{array}$ & $\begin{array}{c}\text { Other drivers } \\
\text { and their } \\
\text { passengers }\end{array}$ \\
\hline & & BMDA & BMDB & BMDC & BMDD & BMDE & BMDF & BMDG & $\mathrm{BMDH}$ & BMDI \\
\hline 2002 & & 302,605 & 34,689 & 3,431 & 35,976 & 263,198 & 38,784 & 17,107 & 28,353 & 218,361 \\
\hline 2003 & & 290,607 & 31,988 & 3,508 & 33,707 & 253,392 & 36,405 & 17,033 & 28,411 & 208,758 \\
\hline 2004 & & 280,840 & 31,000 & 3,221 & 31,130 & 246,489 & 34,881 & 16,648 & 25,641 & 203,670 \\
\hline 2005 & & 271,017 & 28,126 & 3,201 & 28,954 & 238,862 & 33,281 & 16,561 & 24,824 & 196,351 \\
\hline 2006 & & 258,404 & 25,523 & 3,172 & 28,673 & 226,559 & 30,982 & 16,196 & 23,326 & 187,900 \\
\hline 2007 & & 247,780 & 23,807 & 2,943 & 27,777 & 217,060 & 30,191 & 16,195 & 23,459 & 177,935 \\
\hline 2008 & & 230,905 & 21,996 & 2,538 & 26,034 & 202,333 & 28,482 & 16,297 & 21,550 & 164,576 \\
\hline \multirow[t]{4}{*}{2007} & Q1 & 58983 & 5,242 & 682 & 6,451 & 51,850 & 7,579 & 3,169 & 4,641 & 43,594 \\
\hline & Q2 & 62,188 & 6,557 & 720 & 7,022 & 54,446 & 7,193 & 4,498 & 6,459 & 44,038 \\
\hline & Q3 & 63,562 & 6,516 & 772 & 7,338 & 55,452 & 7,154 & 4,706 & 6,917 & 44,785 \\
\hline & Q4 & 63,047 & 5,492 & 769 & 6,966 & 55,312 & 8,265 & 3,822 & 5,442 & 45,518 \\
\hline \multirow[t]{4}{*}{2008} & Q1 & 56466 & 4,860 & 598 & 6,210 & 49,658 & 7,463 & 3,199 & 4,240 & 41,564 \\
\hline & Q2 & 56,837 & 5,951 & 586 & 6,645 & 49,606 & 6,835 & 4,477 & 5,907 & 39,618 \\
\hline & Q3 & 57,829 & 5,956 & 661 & 6,704 & 50,464 & 6,338 & 4,826 & 6,345 & 40,320 \\
\hline & Q4 & 59,773 & 5,229 & 693 & 6,475 & 52,605 & 7,846 & 3,795 & 5,058 & 43,074 \\
\hline \multirow[t]{3}{*}{2009} & $\mathrm{Q} 1^{2}$ & 50550 & 4,330 & 550 & 5,460 & 44,500 & 6,740 & 3,110 & 3,800 & 36,900 \\
\hline & $\mathrm{Q} 2^{2}$ & 55280 & 5,660 & 570 & 6,590 & 48,150 & 6,500 & 4,870 & 6,020 & 37,890 \\
\hline & $\mathrm{Q}^{2}$ & 57360 & 6,060 & 610 & 6,520 & 50,230 & 6,220 & 5,170 & 6,190 & 39,780 \\
\hline
\end{tabular}

1 Includes riders and passengers of mopeds, motor scooters and combinations.

2 Provisional.

Source: Department for Transport: 02078906595 


\begin{tabular}{|c|c|c|c|c|c|c|c|c|c|c|}
\hline \multicolumn{2}{|c|}{ Table 6.12} & \multicolumn{9}{|c|}{ Local (stage) bus services: fare indices } \\
\hline \multicolumn{2}{|c|}{ Great Britain } & & & & & & & & & $1995=100$ \\
\hline & & London & $\begin{array}{l}\text { English } \\
\text { metropolitan } \\
\text { areas }\end{array}$ & $\begin{array}{c}\text { English shire } \\
\text { counties }\end{array}$ & England & Scotland & Wales & $\begin{array}{c}\text { All Great } \\
\text { Britain }\end{array}$ & $\begin{array}{l}\text { All outside } \\
\text { London }\end{array}$ & $\begin{array}{l}\text { All outside } \\
\text { London and } \\
\text { English } \\
\text { metropolitan } \\
\text { areas } \\
\end{array}$ \\
\hline \multicolumn{2}{|c|}{$2001 / 02^{1}$} & 115.5 & 137.4 & 135.1 & 130.3 & 131.8 & 133.5 & 130.6 & 135.3 & 134.4 \\
\hline \multicolumn{2}{|c|}{$2002 / 03^{1}$} & 114.8 & 142.7 & 141.7 & 134.2 & 134.5 & 139.5 & 134.5 & 140.8 & 139.9 \\
\hline \multicolumn{2}{|c|}{$2003 / 04^{1}$} & 116.9 & 148.0 & 148.5 & 139.1 & 136.8 & 145.5 & 139.1 & 146.3 & 145.4 \\
\hline \multicolumn{2}{|c|}{$2004 / 05^{1}$} & 126.8 & 154.2 & 155.7 & 146.2 & 140.4 & 152.4 & 145.7 & 152.5 & 151.4 \\
\hline \multicolumn{2}{|c|}{$2005 / 06^{1}$} & 139.7 & 167.0 & 165.9 & 159.2 & 144.6 & 160.2 & 156.3 & 162.4 & 160.3 \\
\hline \multicolumn{2}{|c|}{$2006 / 07^{1}$} & 151.5 & 168.3 & 158.8 & 156.1 & 151.5 & 167.0 & 155.7 & 160.3 & 170.5 \\
\hline \multicolumn{2}{|c|}{$2007 / 08^{1}$} & 159.5 & 177.4 & 168.1 & 163.9 & 154.1 & 172.9 & 162.9 & 168.6 & 180.1 \\
\hline \multicolumn{2}{|c|}{$2008 / 09^{1}$} & 160.2 & 188.8 & 178.8 & 173.5 & 164.2 & 186.4 & 172.8 & 179.5 & 186.5 \\
\hline \multirow{3}{*}{2005} & & BAKG & BAKH & BAKI & BAKJ & BAKK & BAKL & BAKM & BAKN & BAKO \\
\hline & $\mathrm{Q}^{2}$ & 137.7 & 164.4 & 162.6 & 153.2 & 142.8 & 155.7 & 152.1 & 159.7 & 160.8 \\
\hline & Q4 & 131.7 & 166.4 & 166.8 & 154.1 & 144.2 & 158.3 & 153.1 & 162.8 & 158.8 \\
\hline \multirow[t]{4}{*}{2006} & Q1 & 148.7 & 173.1 & 171.3 & 162.3 & 147.6 & 160.9 & 160.6 & 167.6 & 171.4 \\
\hline & Q2 & 148.7 & 166.2 & 155.6 & 153.3 & 150.7 & 166.1 & 153.2 & 157.9 & 167.9 \\
\hline & $\mathrm{Q}^{2}$ & 147.7 & 166.4 & 157.1 & 153.8 & 150.7 & 166.3 & 153.8 & 158.8 & 167.4 \\
\hline & Q4 & 145.7 & 167.1 & 160.7 & 155.0 & 151.6 & 166.4 & 154.9 & 161.0 & 166.7 \\
\hline \multirow[t]{4}{*}{2007} & Q1 & 164.0 & 173.4 & 161.8 & 162.2 & 152.7 & 169.3 & 161.1 & 163.7 & 180.0 \\
\hline & Q2 & 164.0 & 175.6 & 164.0 & 163.9 & 154.1 & 172.9 & 162.9 & 165.7 & 181.3 \\
\hline & Q3 & 163.1 & 176.0 & 167.5 & 165.7 & 153.7 & 175.4 & 164.6 & 167.8 & 181.3 \\
\hline & Q4 & 154.4 & 176.2 & 169.2 & 164.5 & 154.8 & 178.1 & 163.8 & 169.1 & 177.0 \\
\hline \multirow[t]{4}{*}{2008} & Q1 & 156.4 & 181.6 & 171.5 & 167.4 & 155.6 & 178.8 & 166.4 & 171.9 & 180.9 \\
\hline & Q2 & 156.4 & 183.7 & 174.4 & 169.1 & 160.5 & 184.5 & 168.6 & 175.0 & 181.8 \\
\hline & Q3 & 157.1 & 187.3 & 178.8 & 172.1 & 165.2 & 186.3 & 171.7 & 179.2 & 184.0 \\
\hline & Q4 & 158.5 & 189.7 & 180.7 & 173.9 & 165.6 & 187.5 & 173.3 & 180.9 & 185.9 \\
\hline \multirow[t]{4}{*}{2009} & Q1 & 168.8 & 196.0 & 182.3 & 179.5 & 170.1 & 190.0 & 178.8 & 184.4 & 195.2 \\
\hline & Q2 & 168.8 & 197.8 & 183.9 & 180.6 & 175.2 & 194.3 & 180.5 & 186.8 & 196.0 \\
\hline & Q3 & 168.8 & 197.8 & 184.7 & 181.0 & 175.3 & 195.3 & 180.8 & 187.2 & 196.1 \\
\hline & Q4 & 168.8 & 198.9 & 185.2 & 181.5 & 176.0 & 195.3 & 181.3 & 187.9 & 196.5 \\
\hline
\end{tabular}

1 Owing to rounding financial year data may differ slightly from that published by DfT.

2 London bus fares reduced overall in Q3 owing to TfL's free travel scheme for children aged 11 to 15 , introduced in September 2005 and students aged 16 \& 17 in September 2006.

Source: Department for Transport: 02079444139

\begin{tabular}{|c|c|c|c|c|c|c|c|}
\hline \multicolumn{2}{|c|}{ Table 6.13} & \multicolumn{6}{|c|}{ National Rail and London Underground ${ }^{1}$} \\
\hline & & \multicolumn{6}{|r|}{ Millions } \\
\hline & & \multicolumn{3}{|c|}{ National Rail: passenger kilometres by ticket type } & \multicolumn{3}{|c|}{ London Underground: passenger journeys ${ }^{5,6}$} \\
\hline & & Ordinary fares & Season tickets & Total $^{4}$ & $\begin{array}{c}\text { Full and } \\
\text { reduced fares }\end{array}$ & Season tickets & Total \\
\hline $2002 / 0$ & & 28,394 & 11,284 & 39,678 & 495 & 446 & 942 \\
\hline $2003 / 0$ & & 28,945 & 11,967 & 40,911 & 491 & 457 & 948 \\
\hline $2004 / 0$ & & 29,390 & 12,372 & 41,762 & 486 & 490 & 976 \\
\hline $2005 / 0$ & & 30,011 & 13,200 & 43,211 & 460 & 510 & 970 \\
\hline $2006 / 0$ & & 32,930 & 13,288 & 46,218 & 485 & 513 & 998 \\
\hline $2007 / 0$ & & 33,921 & 15,087 & 49,008 & 581 & 515 & 1,096 \\
\hline \multicolumn{2}{|c|}{$2008 / 09$} & 35,234 & 15,463 & 50,697 & 616 & 473 & 1,089 \\
\hline \multirow{3}{*}{2006} & & BMGB & BMGD & BMGA & BMGF & BMGG & BMGE \\
\hline & Q3 & 8,580 & 2,954 & 11,534 & 125 & 125 & 250 \\
\hline & Q4 & 8,424 & 3,412 & 11,837 & 128 & 129 & 256 \\
\hline \multirow[t]{4}{*}{2007} & Q1 & 7,883 & 3,786 & 11,669 & 125 & 116 & 241 \\
\hline & Q2 & 8,299 & 3,606 & 11,904 & 130 & 118 & 248 \\
\hline & Q3 & 8,846 & 3,512 & 12,358 & 139 & 117 & 256 \\
\hline & $\mathrm{Q}^{2,3}$ & 8,452 & 3,969 & 12,421 & 140 & 123 & 263 \\
\hline \multirow[t]{4}{*}{2008} & Q1 & 8,324 & 4,001 & 12,324 & 140 & 111 & 251 \\
\hline & Q2 & 9,011 & 3,856 & 12,776 & 142 & 107 & 249 \\
\hline & Q3 & 9,264 & 3,620 & 12,793 & 151 & 113 & 264 \\
\hline & Q4 & 8,674 & 3,963 & 12,548 & 135 & 101 & 236 \\
\hline \multirow[t]{4}{*}{2009} & Q1 & 8,286 & 4,026 & 12,311 & 135 & 101 & 236 \\
\hline & Q2 & 8,838 & 3,570 & 12,408 & 145 & 101 & 246 \\
\hline & Q3 & 9,331 & 3,353 & 12,684 & 141 & 93 & 234 \\
\hline & Q4 & 9,137 & 3,740 & 12,877 & 156 & 102 & 258 \\
\hline
\end{tabular}

1 For passenger kilometres, there is a break in series due to new metholology which has been applied to the 2003-04 and 2007-08 data to improve the categorisations of ticket type. Therefore direct comparisons between 2007-08 and earlier years should not be made. 2 Data for 2007 Q4 includes new TOCs as at 11 November 2007. New TOCs include Arriva Cross Country, East Midlands Trains, London Midland, London Overground and National.

3 TOCs that ceased to exist from 11 November 2007 include Silverlink (METRO and Country), Central Trains, Virgin Cross Country, Midland Main Line and GNER.

4 These data do not include the non-franchised train operating companies.

5 Revisions have been made to the Ordinary fares and Season tickets quarterly and annual data from 2003-04 to 2006-07. These revisions have been provided by ATOC

6 These data do not include the non-franchised train operating companies.

Source: Office of Rail Regulation: www.rail-reg.gov.uk, Tel 02072820110 Department for Transport: 02079443076 


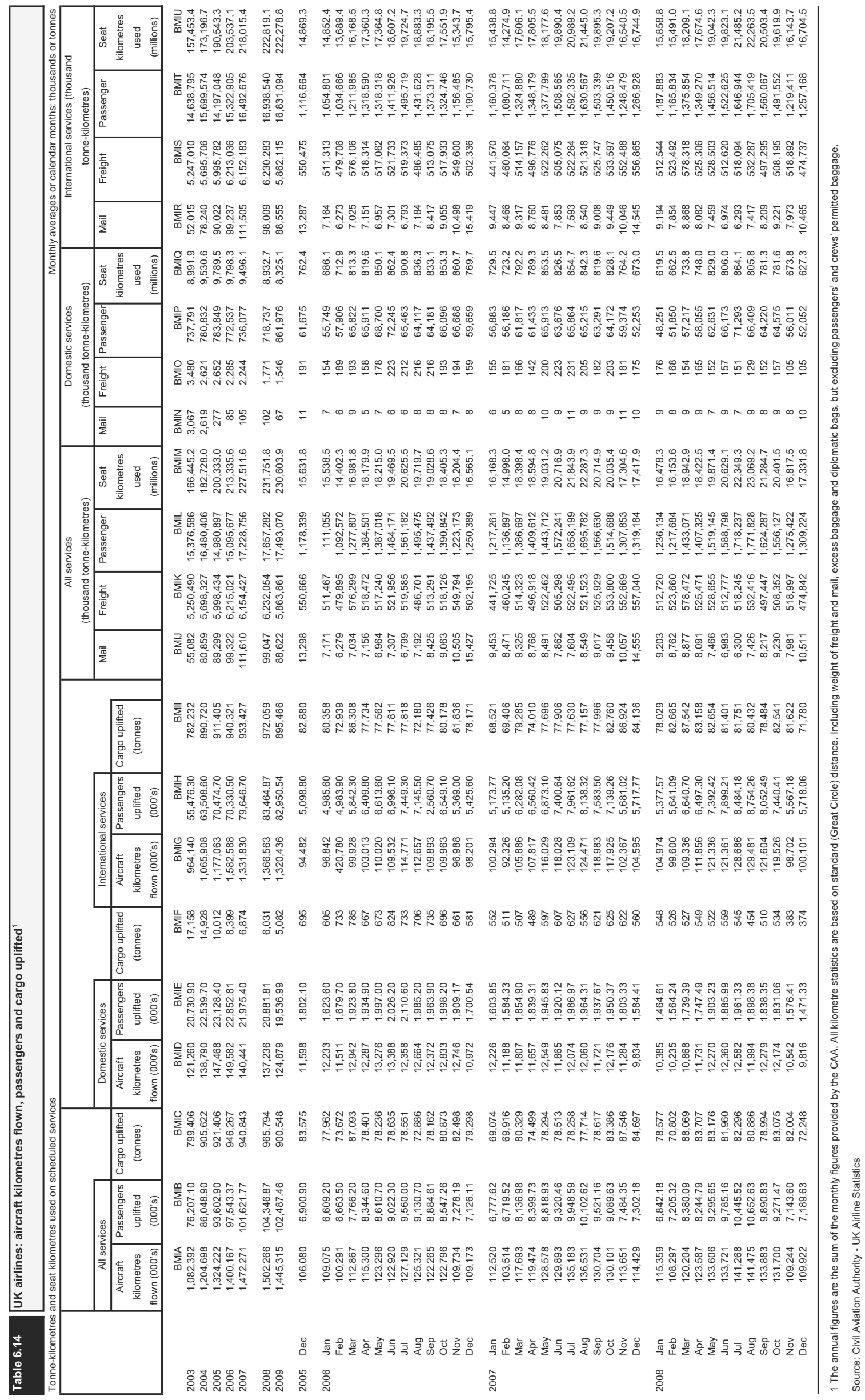




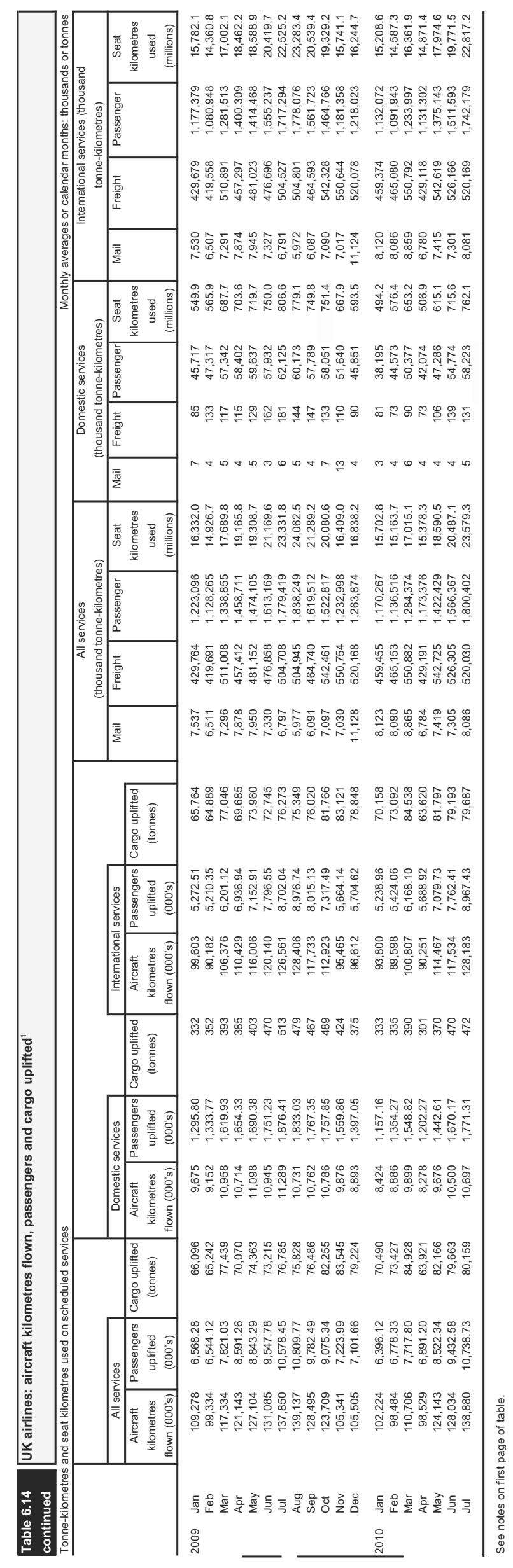




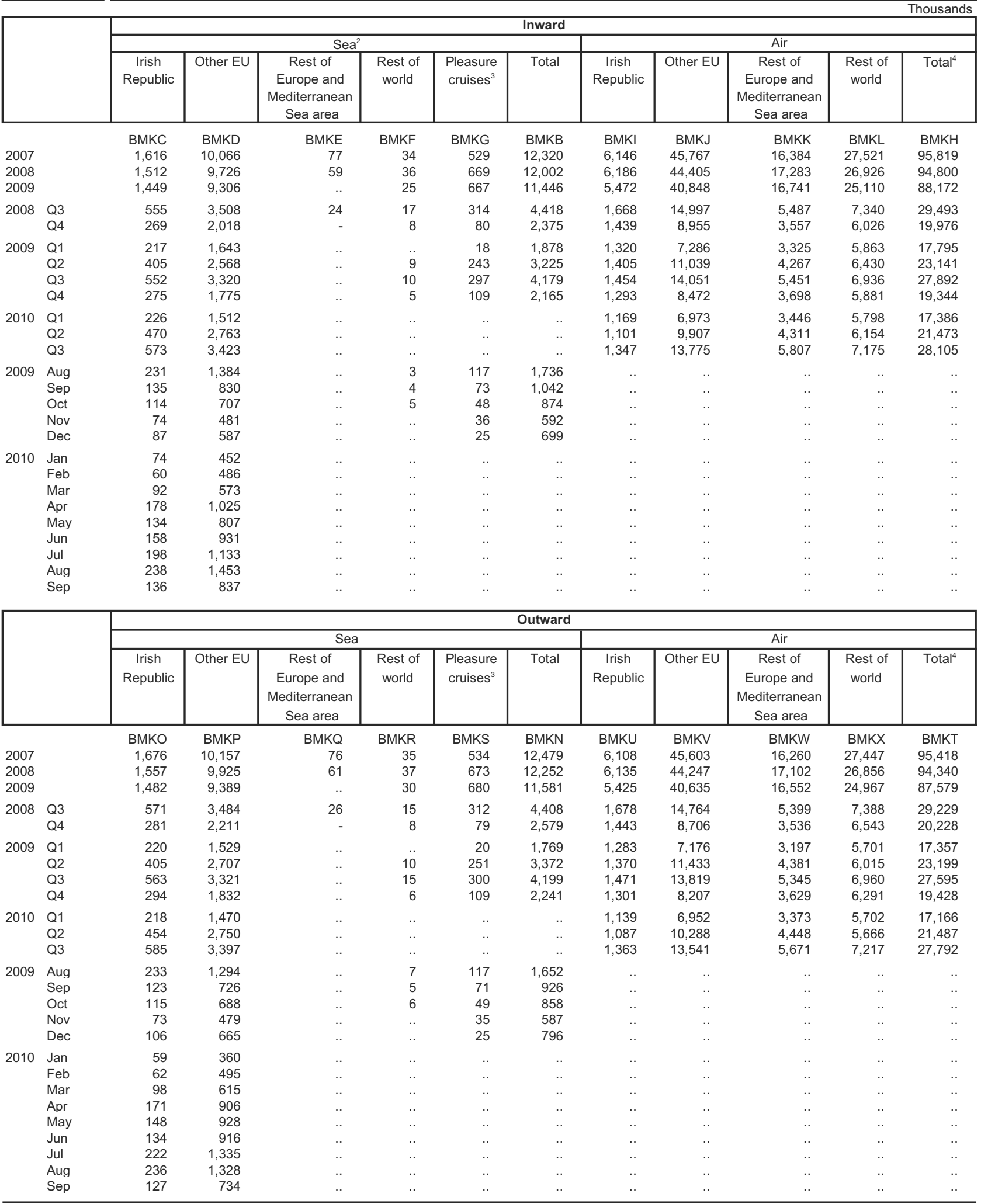

Note: Sea and Air passenger numbers are seasonal, which should be taken into account when comparing figures within a year.

1 Excluding movement by land across the frontier between the Irish Republic and Northern Ireland, passengers travelling between the Channel Islands and Great Britain, passengers carried in aircraft chartered by British government departments and as far as possible, passengers travelling by sea on day trips and $\mathrm{HM}$ and other Armed Forces travelling in the course of their duties.

2 Due to rounding methods, total annual data may not equal the quarterly totals.

3 Passengers on pleasure cruises beginning and/or ending at UK seaports (excluding scheduled voyages between Southampton and New York

which are included in rest of world).

4 Excluding oil rigs.

Source: Department for Transport, Tel: 02079444131

Civil Aviation Authority 


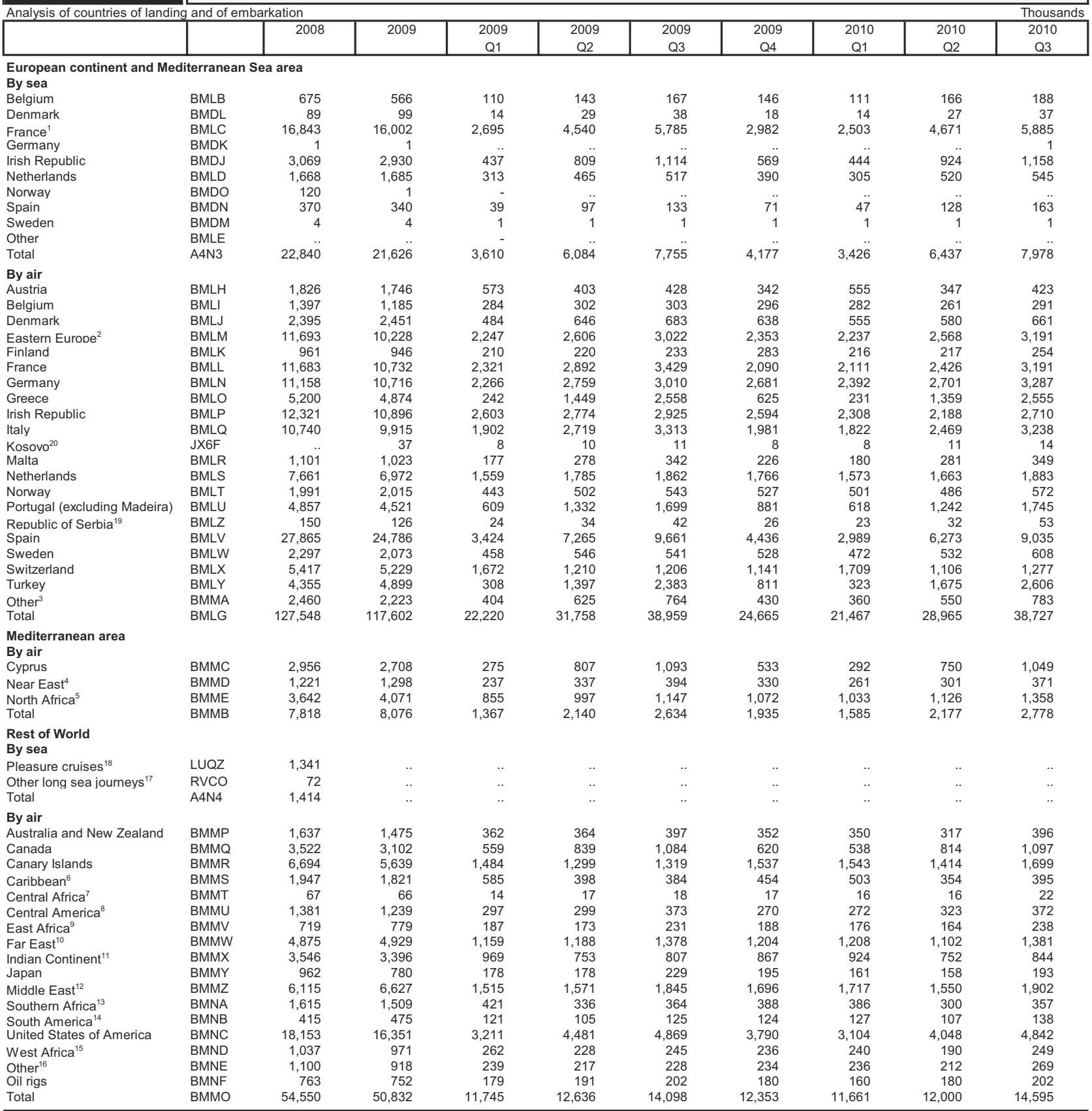

Note: Sea and Air passenger numbers are seasonal, which should be taken into account when comparing figures within a year.

1 Includes hovercraft passengers.

2 Including Albania, Armenia, Azerbaijan, Bulgaria, Czech Republic, Hungary, Kyrgyzstan, Poland, Republic of Moldova, Tajikistan, Turkmenistan, Romania and Commonwealth of Independent States.

4 Including Jordan, Lebanon, Israel and Syria.

5 Including Algeria, Egypt, Libya, Morocco and Tunisia.

6 Including Bahamas, Barbados, Bermuda, Cayman Islands, French Antilles, Jamaica, Leeward Islands, Netherlands Antilles, Puerto Rico, Trinidad and Tobago, Turks and Caicos Islands, US Virgin Islands and Windward Islands.

7 Including Angola, Central African Republic, Chad, Congo, Democratic Republic of Congo, Malawi and Zambia.

8 Including Belize, Costa Rica, Cuba, Dominican Republic, El Salvador, Guatemala, Haiti, Honduras, Mexico, Nicaraqua and Panama.

8 Inchang Burze, Costa Rica, Cuba, Domican Repubic, El Salvador

10 Including Bandar Seri Begawan, Burma, China, Hong Kong, Indonesia, Kampuchea, Korea, Laos, Malaysia, Nepal, Philippines, Singapore, Taiwan, Thailand and Vietnam.

1 Including Afghanistan, Bangladesh India, Pakistan and Sri Lanka.

12 Including Bahrain, Iran, Iraq, Kuwait, Persian Gulf States, Qatar, Republic of North Yemen, Republic of South Yemen, Saudi Arabia and United Arab Emirates.

13 Including Botswana, Lesotho, Mozambique, Namibia, South African Republic, Swaziland and Zimbabwe.

14 Including Argentina, Bolivia, Brazil, Chile, Colombia, Ecuador, Guyana, Paraquay, Peru, Uruquav and Venezuela.

15 Including Benin, Cameroon, Equatorial Guinea, Gabon, Gambia, Ghana, Guinea, Guinea Bissau, Ivory Coast, Liberia, Mali, Mauritania, Niger, Nigeria, Senegal, Sierra Leone, Togo, Upper Volta and Western Sahara.

16 Atlantic Ocean Islands, Indian Ocean Islands and Pacific Ocean Islands and Madeira.

17 Australia and New Zealand, Africa, Caribbean and other areas of the world (excluding USA and Canada).

18 Passengers on pleasure cruises beginning and/or ending at UK seaports (excluding scheduled voyages between Southampton and New York which are included in Other sea journeys).

19 Was originally Yugoslavia, then 1990 renamed Serbia and Montenegro, then 2006 became Republic of Serbia an independant state.

20 Final annual total for 2008 not available. The UN did not regard Kosovo as a country in its own right during the 1st quarter 2008.

Source: Department for Transport;

Civil Aviation Authority 